\title{
Commuting involutions whose fixed point set consists of two special components
}

\author{
by \\ Pedro L. Q. Pergher (São Carlos) and \\ Rogério de Oliveira (Três Lagoas)
}

\begin{abstract}
Let $F^{n}$ be a connected, smooth and closed $n$-dimensional manifold. We call $F^{n}$ a manifold with property $\mathcal{H}$ when it has the following property: if $N^{m}$ is any smooth closed $m$-dimensional manifold with $m>n$ and $T: N^{m} \rightarrow N^{m}$ is a smooth involution whose fixed point set is $F^{n}$, then $m=2 n$. Examples of manifolds with this property are: the real, complex and quaternionic even-dimensional projective spaces $R P^{2 n}$, $C P^{2 n}$ and $H P^{2 n}$, and the connected sum of $R P^{2 n}$ and any number of copies of $S^{n} \times S^{n}$, where $S^{n}$ is the $n$-sphere and $n$ is not a power of 2 . In this paper we describe the equivariant cobordism classification of smooth actions $\left(M^{m} ; \Phi\right)$ of the group $Z_{2}^{k}$ on closed smooth $m$-dimensional manifolds $M^{m}$ for which the fixed point set of the action consists of two components $K$ and $L$ with property $\mathcal{H}$, and where $\operatorname{dim}(K)<\operatorname{dim}(L)$. The description is given in terms of the set of equivariant cobordism classes of involutions fixing $K \cup L$.
\end{abstract}

1. Introduction. A natural question in equivariant cobordism is the classification, up to cobordism, of smooth $Z_{2}^{k}$-actions $\left(M^{m} ; \Phi\right)$, defined on closed and smooth $m$-dimensional manifolds $M^{m}$, with a given condition on the fixed data of $\Phi$. Here, $Z_{2}^{k}$ is considered as the group generated by $k$ commuting involutions $T_{1}, \ldots, T_{k}$, and the fixed data of $\Phi$ is $\eta=\bigoplus_{\varrho} \varepsilon_{\varrho} \rightarrow F$, where $F$ is the fixed point set of $\Phi$ and $\eta=\bigoplus_{\varrho} \varepsilon_{\varrho}$ is the normal bundle of $F$ in $M^{m}$ decomposed into eigenbundles $\varepsilon_{\varrho}$ with $\varrho$ running through the $2^{k}-1$ nontrivial irreducible representations of $Z_{2}^{k}$. For example, see [12] $(F=$ real projective space $R P^{2 n}$ and $k=1$ ), [3] ( $F$ with constant dimension $n$, $\operatorname{dim}(\eta) \geq n$ and $k=1)$, [2, Section 31] ( $F=$ a set of isolated points and $k=2),[10](F=$ union of two real projective spaces and $k=1)$ and [5] ( $F$ $=$ a connected $n$-dimensional manifold, $\operatorname{dim}(\eta) \geq\left(2^{k}-1\right) n$ and any $\left.k\right)$.

2000 Mathematics Subject Classification: Primary 57R85; Secondary 57R75.

Key words and phrases: $Z_{2}^{k}$-action, fixed data, property $\mathcal{H}$, simultaneous cobordism, equivariant cobordism, characteristic number, projective bundle.

The first author was partially supported by CNPq and FAPESP. 
An interesting feature of this question is that, in some cases, the classification for $k=1$ completely determines the corresponding classification for any $k \geq 1$. For example, this happens when $F=R P^{2 n}, C P^{2 n}, H P^{2 n}$ or $Q P^{2}$, the real, complex or quaternionic even-dimensional projective space, or the Cayley projective plane (see [12] and [1]); this also happens when $F=V^{n} \cup\{p\}$, where $p$ is a point and $V^{n}$ is any $n$-dimensional connected manifold with $n>0$ (see [6]). In all these cases, the equivariant cobordism classes of $Z_{2}^{k}$-actions fixing $F$ can be represented by a special set of $Z_{2}^{k}$-actions obtained from involutions fixing $F$.

More precisely, this can be placed in the following general setting. Let $(W ; T)$ be any involution. For each $r$ with $1 \leq r \leq k$, one may construct a special action of $Z_{2}^{k}$ on the product $W^{2^{r-1}}=W \times \cdots \times W\left(2^{r-1}\right.$ factors), which we denote by $\Gamma_{r}^{k}(W ; T)$, in the following inductive way. First set $\Gamma_{1}^{1}(W ; T)=(W ; T)$. Taking $k \geq 2$ and supposing by induction that one has constructed $\Gamma_{k-1}^{k-1}(W ; T)$, define $\Gamma_{k}^{k}(W ; T)=\left(W^{2^{k-1}} ; T_{1}, \ldots, T_{k}\right)$, where $\left(W^{2^{k-1}} ; T_{1}, \ldots, T_{k-1}\right)=\left(W^{2^{k-2}} \times W^{2^{k-2}} ; T_{1}, \ldots, T_{k-1}\right)=\Gamma_{k-1}^{k-1}(W ; T) \times$ $\Gamma_{k-1}^{k-1}(W ; T)$ and $T_{k}$ acts by switching $W^{2^{k-2}} \times W^{2^{k-2}}$. This defines $\Gamma_{k}^{k}(W ; T)$ for any $k \geq 1$. Next, define $\Gamma_{r}^{k}(W ; T)=\left(W^{2^{r-1}} ; T_{1}, \ldots, T_{k}\right)$ by setting $\left(W^{2^{r-1}} ; T_{1}, \ldots, T_{r}\right)=\Gamma_{r}^{r}(W ; T)$ and letting $T_{r+1}, \ldots, T_{k}$ act trivially. If $F$ is a connected manifold, one has the twist involution $t: F \times F \rightarrow F \times F$, given by $t(x, y)=(y, x)$, and $\Gamma_{r}^{k}(F \times F ; t)=\left(F^{2^{r}} ; T_{1}, \ldots, T_{k}\right)$, where $\left(T_{1}, \ldots, T_{r}\right)$ is the usual twist $Z_{2}^{r}$-action on $F^{2^{r}}$ which interchanges factors and $T_{r+1}, \ldots, T_{k}$ act trivially. In this special case, we allow $r$ to be zero, setting $\Gamma_{0}^{k}(F \times F ; t)=\left(F ; T_{1}, \ldots, T_{k}\right)$, where each $T_{i}$ is the identity involution.

Now, from a given $Z_{2}^{k}$-action $(M ; \Phi), \Phi=\left(T_{1}, \ldots, T_{k}\right)$, we can obtain a collection of new $Z_{2}^{k}$-actions, described as follows: first, each automorphism $\sigma: Z_{2}^{k} \rightarrow Z_{2}^{k}$ yields a new action given by $\left(M ; \sigma\left(T_{1}\right), \ldots, \sigma\left(T_{k}\right)\right)$; we denote this action by $\sigma(M ; \Phi)$. Next, it was shown in [7] that if $(M ; \Phi)$ has fixed data $\bigoplus_{\varrho} \varepsilon_{\varrho} \rightarrow F$ and one of the eigenbundles $\varepsilon_{\theta}$ is isomorphic to $\varepsilon_{\theta}^{\prime} \oplus R$, where $R \rightarrow F$ is the trivial one-dimensional bundle, then there is an action $(N ; \Psi)$ with fixed data $\bigoplus_{\varrho} \mu_{\varrho} \rightarrow F$, where $\mu_{\varrho}=\varepsilon_{\varrho}$ if $\varrho \neq \theta$ and $\mu_{\theta}=\varepsilon_{\theta}^{\prime}$. Thus, the iterated process of removing sections may possibly enlarge the set $\left\{\sigma(M ; \Phi) \mid \sigma \in \operatorname{Aut}\left(Z_{2}^{k}\right)\right\}$.

Summarizing, from a given involution $(W ; T)$, we obtain a collection of $Z_{2}^{k}$-actions by applying the operations $\sigma \Gamma_{r}^{k}$ to $(W ; T)$ and next by removing the (possible) sections from the resulting eigenbundles. If $(W ; T)$ and $(N ; S)$ are $Z_{2}$-cobordant, then the actions obtained by removing the same sections from $\sigma \Gamma_{r}^{k}(W ; T)$ and $\sigma \Gamma_{r}^{k}(N ; S)$ are $Z_{2}^{k}$-cobordant, and the converse is also true (it suffices to look at the fixed data; see Section 2$)$. Also, if $(W ; T)$ fixes $F$, then every $Z_{2}^{k}$-action of the above collection fixes $F$. 
Now, for a given $F$, denote by $\mathcal{A}_{k}(F)$ the set of all equivariant cobordism classes of $Z_{2}^{k}$-actions containing a representative $(M ; \Phi)$ with $F$ as fixed point set, and by $\mathcal{A}_{k}^{m}(F) \subset \mathcal{A}_{k}(F)$ the subset of $m$-dimensional classes. Denote by $\mathcal{B}_{k}(F) \subset \mathcal{A}_{k}(F)$ the subset consisting of the classes obtained from $\mathcal{A}_{1}(F)$ through the above procedure, and by $\mathcal{B}_{k}^{m}(F) \subset \mathcal{B}_{k}(F)$ the subset of $m$ dimensional classes. For a fixed partition $\tau=\left\{\tau_{1}, \ldots, \tau_{p}\right\}(p \geq 1)$ of the set of components of $F$, write $F$ as the disjoint union $F=F_{1} \cup \cdots \cup F_{p}$, where each $F_{i}$ is the union of the members of $\tau_{i}$. If $\left[\left(M_{i}^{m} ; \Phi_{i}\right)\right] \in \mathcal{B}_{k}^{m}\left(F_{i}\right)$, $1 \leq i \leq p$, then $\bigcup_{i=1}^{p}\left(M_{i}^{m} ; \Phi_{i}\right)$ represents a class of $\mathcal{A}_{k}^{m}(F)$; denote by $\mathcal{P}_{k, \tau}^{m}(F) \subset \mathcal{A}_{k}^{m}(F)$ the subset consisting of the classes obtained in this way, and set

$$
\mathcal{P}_{k}(F)=\bigcup_{m} \bigcup_{\tau} \mathcal{P}_{k, \tau}^{m}(F) \subset \mathcal{A}_{k}(F) .
$$

Then one looks, in general, for those $F$ for which $\mathcal{A}_{k}(F)=\mathcal{P}_{k}(F)$ (this makes precise the statement "the classification for $k=1$ completely determines the corresponding classification for any $k \geq 1$ "). Under this setting, the above mentioned results say that this is true for $F=V^{n} \cup\{p\}, R P^{2 n}, C P^{2 n}, H P^{2 n}$ and $Q P^{2}$ (if $F$ is connected, then $\mathcal{P}_{k}(F)=\mathcal{B}_{k}(F)$; in the case $F=V^{n} \cup\{p\}$, also $\mathcal{P}_{k}(F)=\mathcal{B}_{k}(F)$, since an involution cannot have precisely one fixed point).

Recently, in [8], we introduced the following concept: given a connected, smooth, closed $n$-dimensional manifold $F^{n}$, we call $F^{n}$ a manifold with property $\mathcal{H}$ if every involution $\left(M^{m} ; T\right)$ fixing $F^{n}$ has $m=n$ or $m=2 n$. This definition was inspired by [2, Theorem 27.7] and [12], where it was shown that $R P^{2 n}$ has this property (similar arguments show that $C P^{2 n}, H P^{2 n}$ and $Q P^{2}$ have this property). The following facts concerning property $\mathcal{H}$ were proved: if $F^{n}$ has property $\mathcal{H}$, then $n$ is even, $F^{n}$ is nonbounding, the tangent bundle over $F^{n}$ does not have sections and $F^{n}$ cannot be the total space of a nontrivial differentiable fibering of closed manifolds. Also, property $\mathcal{H}$ is not a cobordism invariant, but is a homotopy invariant (an intrinsic characterization of these manifolds is still an open question). In addition, we presented a lot of new examples of manifolds with this property (that is, not homotopy equivalent to the known examples $R P^{2 n}, C P^{2 n}, H P^{2 n}$ and $Q P^{2}$ ): all nonbounding 2-dimensional manifolds, all simply connected and nonbounding 4-dimensional manifolds (for example, the connected sum of $C P^{2}$ and any number of copies of $S^{2} \times S^{2}$ ), all nonbounding 8-dimensional manifolds $M^{8}$ with $H^{1}\left(M^{8}, Z_{2}\right)=0$ and $H^{2}\left(M^{8}, Z_{2}\right)=0$, all nonbounding 16-dimensional manifolds $M^{16}$ with $H^{i}\left(M^{16}, Z_{2}\right)=0$ for $1 \leq i \leq 4$, and the connected sum $R P^{2 n} \#\left(S^{n} \times S^{n}\right) \# \cdots \#\left(S^{n} \times S^{n}\right)$ where $n$ is not a power of 2 .

Generalizing [1], where it had been shown that every $Z_{2}^{k}$-action fixing $R P^{2 n}$ is equivariantly cobordant to $\sigma \Gamma_{r}^{k}\left(R P^{2 n} \times R P^{2 n} ; t\right)$ for some 
$\sigma: Z_{2}^{k} \rightarrow Z_{2}^{k}$ and $1 \leq r \leq k$ (again similar arguments, strongly based on the structure of the real $K$-theory of these spaces, work for $C P^{2 n}, H P^{2 n}$ and $Q P^{2}$ ), we showed in [8] that the same result is true for any manifold with property $\mathcal{H}$; in other words, $\mathcal{A}_{k}(F)=\mathcal{P}_{k}(F)=\mathcal{B}_{k}(F)$ for any $F$ with property $\mathcal{H}$.

The objective of this paper is to show that $\mathcal{A}_{k}(F)=\mathcal{P}_{k}(F)$ when $F$ is the disjoint union of two manifolds with property $\mathcal{H}, F=K \cup L$, where $\operatorname{dim}(K)<\operatorname{dim}(L)$. At the end of the paper we give some simple examples of manifolds $F$ for which $\mathcal{A}_{k}(F) \neq \mathcal{P}_{k}(F)$ and of pairs $K, L$ for which $\mathcal{A}_{k}(K \cup L)$ can be explicitly computed from our results.

2. Preliminaries. First we need some basic facts about $Z_{2}^{k}$-actions. Given a $Z_{2}^{k}$-action $(M ; \Phi), \Phi=\left(T_{1}, \ldots, T_{k}\right)$, the fixed point set of $\Phi, F$, is a disjoint union of closed submanifolds of $M$. The normal bundle of $F$ in $M, \eta$, decomposes under $\Phi$ into the Whitney sum of subbundles on which $Z_{2}^{k}$ acts as one of the irreducible (nontrivial) real representations, which are all one-dimensional and can be described by epimorphisms $\varrho: Z_{2}^{k} \rightarrow Z_{2}=$ $\{+1,-1\}: g \in Z_{2}^{k}$ acts on the reals as multiplication by $\varrho(g)$. In other words, $\eta=\bigoplus_{\varrho} \varepsilon_{\varrho}$, where $\varepsilon_{\varrho}$ is the subbundle of $\eta$ on which $Z_{2}^{k}$ acts in the fibers as $\varrho$; that is, where each $T_{j}$ acts as multiplication by $\varrho\left(T_{j}\right)$, and where the sum excludes the trivial homomorphism $1 \in \operatorname{Hom}\left(G, Z_{2}\right)$. Alternatively, $\varepsilon_{\varrho}$ is the normal bundle of $F$ in the fixed point set $F_{\varrho}$ of the subgroup $\operatorname{ker}(\varrho)$.

Setting $\mathcal{P}=\operatorname{Hom}\left(Z_{2}^{k}, Z_{2}\right)-\{1\}$, we can write the fixed data of $(M ; \Phi)$ as $\left(F ;\left\{\varepsilon_{\varrho}\right\}_{\varrho \in \mathcal{P}}\right)$, the fixed set $F$ and a list of $2^{k}-1$ vector bundles over it indexed by $\mathcal{P}$. Each $s$-dimensional component of $\left(F ;\left\{\varepsilon_{\varrho}\right\}_{\varrho \in \mathcal{P}}\right)$ can be considered as an element of $\mathcal{N}_{s}\left(\prod_{\varrho \in \mathcal{P}} B O\left(n_{\varrho}\right)\right)$, the bordism of $s$-dimensional manifolds with a map into a product of classifying spaces $B O\left(n_{\varrho}\right)$ for $n_{\varrho}$-dimensional vector bundles, where $n_{\varrho}$ denotes the dimension of $\varepsilon_{\varrho}$ over the component (this is the simultaneous cobordism between lists of vector bundles: if $\mathcal{P}$ is any finite set, two lists (indexed by $\mathcal{P}$ ) of vector bundles over closed $n$-dimensional manifolds, $\left(F^{n} ;\left\{\varepsilon_{\varrho}\right\}_{\varrho \in \mathcal{P}}\right)$ and $\left(V^{n} ;\left\{\mu_{\varrho}\right\}_{\varrho \in \mathcal{P}}\right)$, are simultaneously cobordant if there exists an $(n+1)$-dimensional manifold $W^{n+1}$ with boundary $\partial\left(W^{n+1}\right)=F^{n} \cup V^{n}$ (disjoint union) and a list of vector bundles over $W^{n+1},\left(W^{n+1} ;\left\{\eta_{\varrho}\right\}_{\varrho \in \mathcal{P}}\right)$, so that each $\eta_{\varrho}$ restricted to $F^{n} \cup V^{n}$ is equivalent to $\left.\varepsilon_{\varrho} \cup \mu_{\varrho}\right)$. According to [11], the equivariant cobordism class of $(M ; \Phi)$ is determined by the simultaneous cobordism class of $\left(F ;\left\{\varepsilon_{\varrho}\right\}_{\varrho \in \mathcal{P}}\right)$.

For example, if $(W ; T)$ is an involution fixing $F$ and if $\eta \rightarrow F$ is the normal bundle of $F$ in $M$, then the fixed data of the $Z_{2}^{k}$-action $\Gamma_{r}^{k}(W ; T)$ described in Section 1 is $\left(F ;\left\{\varepsilon_{\varrho}\right\}_{\varrho \in \mathcal{P}}\right)$, where $\left\{\varepsilon_{\varrho}\right\}_{\varrho \in \mathcal{P}}$ consists of $2^{r-1}$ copies of $\eta \rightarrow F, 2^{r-1}-1$ copies of the tangent bundle $\tau \rightarrow F$ and $2^{k}-2^{r}$ copies of the zero-dimensional bundle $0 \rightarrow F$. In terms of the representations $\varrho \in \mathcal{P}$, $\varepsilon_{\varrho}=0$ when $H=\operatorname{ker}(\varrho)$ does not contain all the involutions $T_{r+1}, \ldots, T_{k}$ 
(equivalently, some $T_{j}$ with $r+1 \leq j \leq k$ acts in $\varepsilon_{\varrho}$ as -1 ), $\varepsilon_{\varrho}=\eta$ when $H$ contains $T_{r+1}, \ldots, T_{k}$ and does not contain $T_{1}$ (equivalently, each $T_{j}$ with $r+1 \leq j \leq k$ acts in $\varepsilon_{\varrho}$ as 1 , and $T_{1}$ acts in $\varepsilon_{\varrho}$ as -1 ), and $\varepsilon_{\varrho}=\tau$ when $H$ contains $T_{1}, T_{r+1}, \ldots, T_{k}$ (equivalently, each $T_{j}$ with $r+1 \leq j \leq k$ and $T_{1}$ act in $\varepsilon_{\varrho}$ as 1). In particular, for a given $F$, the fixed data of the $Z_{2}^{r}$-twist $Z_{2}^{k}$-action $\left(F^{2^{r}} ; T_{1}, \ldots, T_{k}\right)=\Gamma_{r}^{k}(F \times F ; t)$ is $\left(F ;\left\{\varepsilon_{\varrho}\right\}_{\varrho \in \mathcal{P}}\right)$, where $\left\{\varepsilon_{\varrho}\right\}_{\varrho \in \mathcal{P}}$ consists of $2^{r}-1$ copies of the tangent bundle $\tau \rightarrow F$ and $2^{k}-2^{r}$ copies of the zero bundle $0 \rightarrow F$; in terms of $\varrho \in \mathcal{P}, \varepsilon_{\varrho}=\tau$ when each $T_{j}$ with $r+1 \leq j \leq k$ acts in $\varepsilon_{\varrho}$ as 1 , and $\varepsilon_{\varrho}=0$ for the remaining $\varrho \in \mathcal{P}$.

REMARK. Suppose that $\left(F ;\left\{\varepsilon_{\varrho}\right\}_{\varrho \in \mathcal{P}}\right)$ is the fixed data of a $Z_{2}^{k}$-action $(M ; \Phi)$. Denote by $\mathcal{A}$ the set of vector bundles over $F$ which lie in $\left\{\varepsilon_{\varrho}\right\}$. Then $\left(F ;\left\{\varepsilon_{\varrho}\right\}\right)$ gives a map $\theta: \mathcal{P} \rightarrow \mathcal{A}$, and if $\sigma: Z_{2}^{k} \rightarrow Z_{2}^{k}$ is an automorphism, then $\sigma(M ; \Phi)$ gives rise to a new map $\mathcal{P} \rightarrow \mathcal{A}$ which is $\theta$ composed with some bijection $\mathcal{P} \rightarrow \mathcal{P}$. We note that not every bijection $\mathcal{P} \rightarrow \mathcal{P}$ gives a map $\mathcal{P} \rightarrow \mathcal{A}$ which is derived from some automorphism $Z_{2}^{k} \rightarrow Z_{2}^{k}$, since the number of such bijections may be greater than the number of bases of $Z_{2}^{k}$. In particular, we cannot in principle guarantee that all such maps $\mathcal{P} \rightarrow \mathcal{A}$ come from $Z_{2}^{k}$-actions. This is not the case, however, when $k=2$; if $\left(F ;\left\{\varepsilon_{\varrho_{1}}, \varepsilon_{\varrho_{2}}, \varepsilon_{\varrho_{3}}\right\}\right)$ is the fixed data of a $Z_{2}^{2}$-action with map $\mathcal{P}=\left\{\varrho_{1}, \varrho_{2}, \varrho_{3}\right\} \rightarrow \mathcal{A}$, then all the other possible maps $\mathcal{P} \rightarrow \mathcal{A}$ come from $Z_{2}^{2}$-actions, since they are derived from automorphisms $Z_{2}^{2} \rightarrow Z_{2}^{2}$. Therefore all the assertions made in this paper concerning $Z_{2}^{2}$-actions will be independent of the map $\mathcal{P} \rightarrow \mathcal{A}$.

3. Individual cobordism of the bundles of the fixed data. Suppose that $K$ and $L$ are two manifolds with property $\mathcal{H}$ and with $\operatorname{dim}(K)<$ $\operatorname{dim}(L)$. As mentioned in Section 1, we are concerned with the cobordism classification of the $Z_{2}^{k}$-actions $(M ; \Phi)$ for which the fixed point set is $K \cup L$. Let $\left(K ;\left\{\varepsilon_{\varrho}\right\}_{\varrho \in \mathcal{P}}\right) \cup\left(L ;\left\{\mu_{\varrho}\right\}_{\varrho \in \mathcal{P}}\right)$ be the fixed data of $\Phi$. Our first goal is the analysis of the individual cobordism of the eigenbundles $\varepsilon_{\varrho}$ and $\mu_{\varrho}$, and in this direction Theorems 3.9 and 3.10 are the central part of this section. In the next section we will be concerned with the final task, the determination of the simultaneous cobordism of the lists $\left\{\varepsilon_{\varrho}\right\}_{\varrho \in \mathcal{P}}$ and $\left\{\mu_{\varrho}\right\}_{\varrho \in \mathcal{P}}$.

We need a lot of preliminary results, and the first five such results (Lemmas 3.1-3.5) are of general nature. Let $(M ; \Phi)$ be a $Z_{2}^{k}$-action with fixed data $\left(F ;\left\{\varepsilon_{\varrho}\right\}_{\varrho \in \mathcal{P}}\right)$, and let $\Omega$ be a subgroup of $\operatorname{Hom}\left(Z_{2}^{k}, Z_{2}\right)$. Our first step will be to show that the part of the fixed data of $(M ; \Phi)$ given by $\left(F ;\left\{\varepsilon_{\varrho}\right\}_{\varrho \in \Omega \cap \mathcal{P}}\right)$ can be realized as the fixed data of some subgroup $G \subset Z_{2}^{k}$ acting (by restriction) on the fixed point set of the restriction of $\Phi$ to some appropriate subgroup $H \subset Z_{2}^{k}$.

First note that there exists a subgroup $G \subset Z_{2}^{k}$ such that the restriction $\operatorname{Hom}\left(Z_{2}^{k}, Z_{2}\right) \rightarrow \operatorname{Hom}\left(G, Z_{2}\right)$ maps $\Omega$ isomorphically onto $\operatorname{Hom}\left(G, Z_{2}\right)$. In 
fact, consider the natural isomorphism $Z_{2}^{k} \rightarrow \operatorname{Hom}\left(\operatorname{Hom}\left(Z_{2}^{k}, Z_{2}\right), Z_{2}\right)$ given by $T \mapsto \varphi_{T}$, where $\varphi_{T}(\varrho)=\varrho(T)$ for any $\varrho \in \operatorname{Hom}\left(Z_{2}^{k}, Z_{2}\right)$. Choose a basis $\left(\varrho_{1}, \ldots, \varrho_{r}, \xi_{1}, \ldots, \xi_{k-r}\right)$ for $\operatorname{Hom}\left(Z_{2}^{k}, Z_{2}\right)$ so that $\left(\varrho_{1}, \ldots, \varrho_{r}\right)$ is a basis for $\Omega$, and consider the basis $\left(T_{1}, \ldots, T_{r}, S_{1}, \ldots, S_{k-r}\right)$ for $Z_{2}^{k}$ which corresponds to the dual basis $\left(\varrho_{1}^{*}, \ldots, \varrho_{r}^{*}, \xi_{1}^{*}, \ldots, \xi_{k-r}^{*}\right)$ of $\operatorname{Hom}\left(\operatorname{Hom}\left(Z_{2}^{k}, Z_{2}\right), Z_{2}\right)$ under the above isomorphism. Evidently, $\left(\varrho_{1}, \ldots, \varrho_{r}, \xi_{1}, \ldots, \xi_{k-r}\right)$ is the dual basis of $\left(T_{1}, \ldots, T_{r}, S_{1}, \ldots, S_{k-r}\right)$. Set $G=\left\langle T_{1}, \ldots, T_{r}\right\rangle$. Since $\varrho_{i}\left(T_{j}\right)=-1$ if $i=j$ and $\varrho_{i}\left(T_{j}\right)=1$ if $i \neq j$, one sees that $\left(\varrho_{1 \mid G}, \ldots, \varrho_{r \mid G}\right)$ is a basis for $\operatorname{Hom}\left(G, Z_{2}\right)$, and thus the restriction maps $\Omega$ isomorphically onto $\operatorname{Hom}\left(G, Z_{2}\right)$. Now set $H=\left\langle S_{1}, \ldots, S_{k-r}\right\rangle, F_{H}=$ the fixed point set of $H$ and $\Psi=$ the restriction of $\Phi$ to $G \times F_{H}$. One then has the following

Lemma 3.1. The fixed data of the $G$-action $\left(F_{H} ; \Psi\right)$ is $\left(F ;\left\{\mu_{\varrho^{\prime}}\right\}_{\varrho^{\prime} \in \mathcal{P}^{\prime}}\right)$, where for each $\varrho^{\prime} \in \mathcal{P}^{\prime}=\operatorname{Hom}\left(G, Z_{2}\right)-\{1\}$ one has $\mu_{\varrho^{\prime}}=\varepsilon_{\varrho}$, where $\varrho$ is the unique element of $\Omega \cap \mathcal{P}$ with $\varrho_{\mid G}=\varrho^{\prime}$. In other words, the fixed data of $G$ acting on the fixed set of $H$ is $F$ with the subbundles $\varepsilon_{\varrho}, \varrho \in \Omega \cap \mathcal{P}$, and in terms of $\mathcal{P}^{\prime}=\operatorname{Hom}\left(G, Z_{2}\right)-\{1\}$, these subbundles are indexed by the restriction $\Omega \cap \mathcal{P} \rightarrow \mathcal{P}^{\prime}$.

Proof. See Lemma 3.1 of [8].

Lemma 3.2. Let $(M ; \Phi)$ be a $Z_{2}^{k}$-action with fixed data $\left(F ;\left\{\varepsilon_{\varrho}\right\}_{\varrho \in \mathcal{P}}\right)$, and take $\varrho_{0} \in \mathcal{P}$. Then there is an automorphism $\sigma: Z_{2}^{k} \rightarrow Z_{2}^{k}$ such that, if the fixed data of $\sigma(M ; \Phi)$ is $\left(F ;\left\{\mu_{\varrho}\right\}_{\varrho \in \mathcal{P}}\right)$, then $\mu_{\varrho_{1}}=\varepsilon_{\varrho_{0}}$, where $\varrho_{1}$ : $Z_{2}^{k} \rightarrow Z_{2}=\{+1,-1\}$ is the representation given by $\varrho_{1}\left(T_{1}\right)=-1$ and $\varrho_{1}\left(T_{i}\right)=0$ for $i \geq 2$.

Proof. Choose $\tau_{2}, \ldots, \tau_{k}$ generating $\operatorname{ker}\left(\varrho_{0}\right)$ and $\tau_{1} \notin \operatorname{ker}\left(\varrho_{0}\right)$. Then the automorphism $\sigma: Z_{2}^{k} \rightarrow Z_{2}^{k}$ defined by $\sigma\left(T_{i}\right)=\tau_{i}, 1 \leq i \leq k$, clearly works.

Again considering a $Z_{2}^{k}$-action $(M ; \Phi)$ with fixed data $\left(F ;\left\{\varepsilon_{\varrho}\right\}_{\varrho \in \mathcal{P}}\right)$, choose $\varrho_{0} \in \mathcal{P}$ and let $P \subset M$ be any component of the fixed point set of $H=\operatorname{ker}\left(\varrho_{0}\right)$. Denote by $F_{P}$ the union of the components of $F$ that are contained in $P$, and take $T \notin H$. Then the involution $(P ; T)$ has $F_{P}$ as fixed point set, with the normal bundle of $F_{P}$ in $P$ being $\varepsilon_{\varrho_{0}} \rightarrow F_{P}$. Each nontrivial homomorphism from $H$ into $Z_{2}$ gives rise to the pair of representations $\theta, \theta^{\prime} \in \mathcal{P}$, given by $\theta=\theta^{\prime}=$ the given homomorphism on $H, \theta(T)=1$ and $\theta^{\prime}(T)=-1$. One may consider the nontrivial homomorphisms from $H$ into $Z_{2}$ as being indexed by the homomorphisms $\theta$, and the part of the fixed data of $(M ; \Phi)$ over $F_{P}$ can be written as $\left(F_{P} ; \varepsilon_{\varrho_{0}},\left\{\varepsilon_{\theta}, \varepsilon_{\theta^{\prime}}\right\}_{\theta}\right)$. Consider the real projective space bundle $R P\left(\varepsilon_{\varrho_{0}}\right) \rightarrow F_{P}$ associated to $\varepsilon_{\varrho_{0}} \rightarrow F_{P}$, and denote by $\xi \rightarrow R P\left(\varepsilon_{\varrho_{0}}\right)$ the line bundle of the double cover $S\left(\varepsilon_{\varrho_{0}}\right) \rightarrow R P\left(\varepsilon_{\varrho_{0}}\right)$, $S\left(\varepsilon_{\varrho_{0}}\right)$ being the sphere bundle of $\varepsilon_{\varrho_{0}}$. Then one has the object

$$
\left(R P\left(\varepsilon_{\varrho_{0}}\right) ; \xi,\left\{\varepsilon_{\theta} \oplus\left(\xi \otimes \varepsilon_{\theta^{\prime}}\right)\right\}_{\theta}\right),
$$


the projective space bundle $R P\left(\varepsilon_{\varrho_{0}}\right)$ and a list of bundles over it formed by the standard line bundle $\xi$ and the bundles $\varepsilon_{\theta} \oplus\left(\xi \otimes \varepsilon_{\theta^{\prime}}\right)$, where we are suppressing bundle maps. If we set $m_{\theta}=\operatorname{dim}\left(\varepsilon_{\theta} \oplus\left(\xi \otimes \varepsilon_{\theta^{\prime}}\right)\right)$ and $j=\operatorname{dim}(P)$, this object represents an element in the bordism group

$$
\mathcal{N}_{j-1}\left(B O(1) \times \prod_{\theta} B O\left(m_{\theta}\right)\right) .
$$

Lemma 3.3. The object $\left(R P\left(\varepsilon_{\varrho_{0}}\right) ; \xi,\left\{\varepsilon_{\theta} \oplus\left(\xi \otimes \varepsilon_{\theta^{\prime}}\right)\right\}_{\theta}\right)$, which is the union of the corresponding objects over each component of $F_{P}$, bounds as an element of $\mathcal{N}_{j-1}\left(B O(1) \times \prod_{\theta} B O\left(m_{\theta}\right)\right)$.

Proof. This follows from the argument outlined in [6, Section 3; pp. 88-90] (or in [5, Section 2; pp. 107-108]), adapted to the situation in which $F_{P}$ may have several components.

Lemma 3.4. Let $F$ be a connected closed manifold, $\varepsilon \rightarrow F$ a vector bundle over $F$ with $\operatorname{dim}(\varepsilon)=\operatorname{dim}(F)$ and $\xi \rightarrow R P(\varepsilon)$ the usual line bundle. Suppose that $\left\{\kappa_{\varrho}\right\}$ and $\left\{\kappa_{\varrho}^{\prime}\right\}$ are lists of vector bundles over $F$, indexed by the same set $\mathcal{Q}$, such that the list $\left(R P(\varepsilon) ; \xi,\left\{\kappa_{\varrho} \oplus\left(\xi \otimes \kappa_{\varrho}^{\prime}\right)\right\}_{\varrho \in \mathcal{Q}}\right)$ is a (simultaneous) boundary. Then the list $\left(F ; \varepsilon,\left\{\kappa_{\varrho}, \kappa_{\varrho}^{\prime}\right\}_{\varrho \in \mathcal{Q}}\right)$ is simultaneously cobordant to $\left(F ; \tau,\left\{\kappa_{\varrho}, \kappa_{\varrho}^{\prime}\right\}_{\varrho \in \mathcal{Q}}\right)$, where $\tau$ is the tangent bundle of $F$.

Proof. The proof is exactly the argument involving characteristic numbers used in the proof of part b) of the lemma of [5, Section 3; p. 108].

LEMmA 3.5. Let $(M ; \Phi)$ be a $Z_{2}^{k}$-action whose fixed point set consists of two components, $K$ and $L$, and let $\left(K ;\left\{\varepsilon_{\varrho}\right\}_{\varrho \in \mathcal{P}}\right) \cup\left(L ;\left\{\mu_{\varrho}\right\}_{\varrho \in \mathcal{P}}\right)$ be the fixed data of $\Phi$. Suppose that, for every $\varrho \in \mathcal{P}, \operatorname{dim}\left(\varepsilon_{\varrho}\right)=\operatorname{dim}(K)($ in particular, $\left.\operatorname{dim}(M)=2^{k} \operatorname{dim}(K)\right)$ and either $\mu_{\varrho}=0$ or $\operatorname{dim}\left(\mu_{\varrho}\right)+\operatorname{dim}(L) \neq$ $\operatorname{dim}\left(\varepsilon_{\varrho}\right)+\operatorname{dim}(K)$. Then the list $\left(K ;\left\{\varepsilon_{\varrho}\right\}_{\varrho \in \mathcal{P}}\right)$ is simultaneously cobordant to $\left(K ;\left\{\tau_{\varrho}\right\}_{\varrho \in \mathcal{P}}\right)$, where, for each $\varrho \in \mathcal{P}, \tau_{\varrho}$ is the tangent bundle of $K$.

Proof. If $\mu_{\varrho}=0$, then $L$ is a component of the fixed point set of $\operatorname{ker}(\varrho)$, and thus the component of $\operatorname{ker}(\varrho)$ containing $K$ does not contain $L$. If $\operatorname{dim}\left(\mu_{\varrho}\right)+\operatorname{dim}(L) \neq \operatorname{dim}\left(\varepsilon_{\varrho}\right)+\operatorname{dim}(K)$, the component of $\operatorname{ker}(\varrho)$ containing $K$ (with dimension $\operatorname{dim}\left(\varepsilon_{\varrho}\right)+\operatorname{dim}(K)=2 \operatorname{dim}(K)$ ) is different from the component of $\operatorname{ker}(\varrho)$ containing $L$ (with dimension $\left.\operatorname{dim}\left(\mu_{\varrho}\right)+\operatorname{dim}(L)\right)$. Thus, for every $\varrho \in \mathcal{P}$, the component of $\operatorname{ker}(\varrho)$ containing $K$ does not contain $L$. By Lemma 3.3, the list $\left(R P\left(\varepsilon_{\varrho}\right) ; \xi,\left\{\varepsilon_{\theta} \oplus\left(\xi \otimes \varepsilon_{\theta^{\prime}}\right)\right\}_{\theta}\right)$ is then a simultaneous boundary for any $\varrho \in \mathcal{P}$. By iteratively applying Lemma $3.42^{k}-1$ times, one gets the result.

Returning to actions $(M ; \Phi)$ with fixed data $\left(K ;\left\{\varepsilon_{\varrho}\right\}_{\varrho \in \mathcal{P}}\right) \cup\left(L ;\left\{\mu_{\varrho}\right\}_{\varrho \in \mathcal{P}}\right)$, where $K$ and $L$ have property $\mathcal{H}$, set $\operatorname{dim}(M)=m$ and $\operatorname{dim}(K)=p<$ $\operatorname{dim}(L)=q$. To ease the notation, for a vector bundle $\theta$ over $K$ (resp. over $L$ ), write $\theta \equiv \eta$ when there exists an involution $(W ; T)$ fixing $K \cup L$ 
and $\theta$ is the normal bundle of $K$ (resp. of $L$ ) in $M$; if $W$ is not connected, we require that all components of $W$ have the same dimension. Also write 0 for the zero bundle over $K$ (resp. over $L$ ) and $\theta \equiv \tau$ when $\theta$ is cobordant to the tangent bundle over $K$ (resp. over $L$ ).

Lemma 3.6. $\left(\varepsilon_{\varrho}, \mu_{\varrho}\right) \equiv(\eta, \eta),(\tau, \tau),(0,0),(\tau, 0)$ and $(0, \tau)$ are the possibilities for the cobordism types of the pairs $\left(\varepsilon_{\varrho}, \mu_{\varrho}\right), \varrho \in \mathcal{P}$.

Proof. For each $\varrho \in \mathcal{P}$, denote by $U_{\varrho}$ and $V_{\varrho}$ the components of the fixed point set of the subgroup $\operatorname{ker}(\varrho)$ containing $K$ and $L$, respectively. Then either $U_{\varrho}=V_{\varrho}$ or $U_{\varrho} \cap V_{\varrho}=\emptyset$. Choose $T \in Z_{2}^{k}-\operatorname{ker}(\varrho)$. In the first case, $\left(U_{\varrho}, T\right)$ is an involution fixing $K \cup L$, which means that $\left(\varepsilon_{\varrho}, \mu_{\varrho}\right) \equiv(\eta, \eta)$. In the second case, $\left(U_{\varrho}, T\right)$ and $\left(V_{\varrho}, T\right)$ are involutions fixing $K$ and $L$ respectively. Since $K$ has property $\mathcal{H}, \operatorname{dim}\left(U_{\varrho}\right)=p$ or $2 p$. If $\operatorname{dim}\left(U_{\varrho}\right)=p$, then $\left(U_{\varrho}, T\right)=$ $(K, \mathrm{Id})$ and $\varepsilon_{\varrho}=0$. If $\operatorname{dim}\left(U_{\varrho}\right)=2 p$, then $\left(U_{\varrho}, T\right)$ is cobordant to $(K \times K ; t)$ and $\varepsilon_{\varrho} \equiv \tau$ (see [3]). Similarly, $\operatorname{dim}\left(V_{\varrho}\right)=q$ or $2 q ; \mu_{\varrho}=0$ when $\operatorname{dim}\left(V_{\varrho}\right)=q$ and $\mu_{\varrho} \equiv \tau$ when $\operatorname{dim}\left(V_{\varrho}\right)=2 q$. Thus, $\left(\varepsilon_{\varrho}, \mu_{\varrho}\right) \equiv(\tau, \tau),(0,0),(\tau, 0)$ or $(0, \tau)$ in the second case (for dimensional reasons, all these types are different, except for $(\eta, \eta)$ and $(\tau, 0)$ when $\operatorname{dim}(L)=2 \operatorname{dim}(K))$.

For $Z_{2}^{2}$-actions with fixed data $\left(K ;\left\{\varepsilon_{\varrho_{1}}, \varepsilon_{\varrho_{2}}, \varepsilon_{\varrho_{3}}\right\}\right) \cup\left(L ;\left\{\mu_{\varrho_{1}}, \mu_{\varrho_{2}}, \mu_{\varrho_{3}}\right\}\right)$, we additionally have the following

Lemma 3.7. Suppose that $\left(\varepsilon_{\varrho_{i}}, \mu_{\varrho_{i}}\right) \equiv(\eta, \eta)$ for at least one $i \in$ $\{1,2,3\}$. Then the only possibilities for the cobordism types of the objects $\left(\left(\varepsilon_{\varrho_{1}}, \varepsilon_{\varrho_{2}}, \varepsilon_{\varrho_{3}}\right),\left(\mu_{\varrho_{1}}, \mu_{\varrho_{2}}, \mu_{\varrho_{3}}\right)\right)$ are, up to permutations, $((\eta, \eta, \tau),(\eta, \eta, \tau))$ and $((\eta, 0,0),(\eta, 0,0))$.

Proof. Using Lemma 3.6 and the facts that $p<q, p+\sum_{i=1}^{3} \operatorname{dim}\left(\varepsilon_{\varrho_{i}}\right)=$ $q+\sum_{i=1}^{3} \operatorname{dim}\left(\mu_{\varrho_{i}}\right)$ and $p+\operatorname{dim}\left(\varepsilon_{\varrho_{i}}\right)=q+\operatorname{dim}\left(\mu_{\varrho_{i}}\right)$ when $\left(\varepsilon_{\varrho_{i}}, \mu_{\varrho_{i}}\right) \equiv(\eta, \eta)$, we can easily show that all the possibilities except the two listed above are impossible for dimensional reasons.

In Lemma 3.6, if $U_{\varrho} \cap V_{\varrho}=\emptyset$ for every $\varrho \in \mathcal{P}$, the argument shows that the possibilities for $\left(\varepsilon_{\varrho}, \mu_{\varrho}\right)$ are $(\tau, \tau),(0,0),(\tau, 0)$ and $(0, \tau)$. We will be first concerned with this case; to do this, recall the following

Lemma 3.8. Suppose $(M ; \Phi), \Phi=\left(T_{1}, \ldots, T_{k}\right)$, is a $Z_{2}^{k}$-action fixing $F$, where $F$ has property $\mathcal{H}$. Then $(M ; \Phi)$ is equivariantly cobordant to $\sigma \Gamma_{r}^{k}(F \times F ; t)$ for some automorphism $\sigma: Z_{2}^{k} \rightarrow Z_{2}^{k}$ and some $0 \leq r \leq k$.

Proof. As mentioned in Section 1, this is the main result of [8].

Returning to our previous notations, one then has the following

TheOREm 3.9. Suppose $U_{\varrho} \cap V_{\varrho}=\emptyset$ for every $\varrho \in \mathcal{P}$. Then there exist $1 \leq r \leq k, 0 \leq s \leq k$ and automorphisms $\sigma, \sigma^{\prime}: Z_{2}^{k} \rightarrow Z_{2}^{k}$ such that $(M ; \Phi)$ is equivariantly cobordant to $\sigma \Gamma_{r}^{k}(K \times K ; t) \cup \sigma^{\prime} \Gamma_{s}^{k}(L \times L ; t)$. 
Proof. Write $M_{K}$ and $M_{L}$ for the components of $M$ containing $K$ and $L$ respectively. Since $\left(M-\left(M_{K} \cup M_{L}\right) ; \Phi\right)$ is a $Z_{2}^{k}$-action without fixed points, the main result of [11] says that $\left(M-\left(M_{K} \cup M_{L}\right) ; \Phi\right)$ bounds as a manifold with $Z_{2}^{k}$-action. Thus we can suppose, without loss of generality, that $M=M_{K} \cup M_{L}$. If $M_{K} \cap M_{L}=\emptyset$, we obtain the desired result by applying Lemma 3.8 to the actions $\left(M_{K} ; \Phi\right)$ and $\left(M_{L} ; \Phi\right)$ (and in this case we can have $r=1$ and $s=0)$. Therefore we can assume that $M=M_{K}=M_{L}$ is connected (and $m>q>p$ ). Since

$$
\operatorname{dim}(K)+\sum_{\varrho \in \mathcal{P}} \operatorname{dim}\left(\varepsilon_{\varrho}\right)=\operatorname{dim}(L)+\sum_{\varrho \in \mathcal{P}} \operatorname{dim}\left(\mu_{\varrho}\right),
$$

the number of bundles $\varepsilon_{\varrho} \equiv \tau$ is $\geq 2$. Let $\Omega$ be the subset of $\operatorname{Hom}\left(Z_{2}^{k}, Z_{2}\right)$ given by $\Omega=\{1\} \cup\left\{\varrho \in \mathcal{P} \mid \varepsilon_{\varrho} \equiv \tau\right\}$.

We assert that $\Omega$ is a subgroup of $\operatorname{Hom}\left(Z_{2}^{k}, Z_{2}\right)$. In fact, take $\varrho_{1}, \varrho_{2} \in \Omega$ with $\varrho_{1} \neq \varrho_{2}$, and suppose $\varepsilon_{\varrho_{3}}=0$, where $\varrho_{3}=\varrho_{1} \varrho_{2}$. Since $\left\{1, \varrho_{1}, \varrho_{2}, \varrho_{3}\right\}$ is a subgroup of $\operatorname{Hom}\left(Z_{2}^{k}, Z_{2}\right)$, by Lemma 3.1 there exist subgroups $G, H \subset$ $Z_{2}^{k}$ with $G$ isomorphic to $Z_{2}^{2}$ and $Z_{2}^{k}=G \oplus H$, so that the fixed data of the $Z_{2}^{2}$-action obtained by letting $G$ act on the fixed point set $F_{H}$ of $H$ is $\left(K ;\left\{\varepsilon_{\varrho_{1}}, \varepsilon_{\varrho_{2}}, \varepsilon_{\varrho_{3}}\right\}\right) \cup\left(L ;\left\{\mu_{\varrho_{1}}, \mu_{\varrho_{2}}, \mu_{\varrho_{3}}\right\}\right)$. Again we can write $F_{H}=$ $F_{K} \cup F_{L}$, where $F_{K}$ and $F_{L}$ are the components of $F_{H}$ containing $K$ and $L$ respectively. If $F_{K} \cap F_{L}=\emptyset$, the $Z_{2}^{2}$-action $\left(F_{K} ; \Phi_{\mid G}\right)$ has fixed data $\left(K ;\left\{\varepsilon_{\varrho_{1}}, \varepsilon_{\varrho_{2}}, \varepsilon_{\varrho_{3}}\right\}\right)$ with $\varepsilon_{\varrho_{1}} \equiv \tau, \varepsilon_{\varrho_{2}} \equiv \tau$ and $\varepsilon_{\varrho_{3}}=0$, thus contradicting Lemma 3.8. Therefore $F_{K}=F_{L}$ and the $Z_{2}^{2}$-action $\left(F_{K} ; \Phi_{\mid G}\right)$ has fixed data $\left(K ;\left\{\varepsilon_{\varrho_{1}}, \varepsilon_{\varrho_{2}}, \varepsilon_{\varrho_{3}}\right\}\right) \cup\left(L ;\left\{\mu_{\varrho_{1}}, \mu_{\varrho_{2}}, \mu_{\varrho_{3}}\right\}\right)$ with at least one $\mu_{\varrho_{i}}$ being nonzero. Because

$$
\begin{aligned}
\operatorname{dim}\left(F_{K}\right) & =\operatorname{dim}(K)+\operatorname{dim}\left(\varepsilon_{\varrho_{1}}\right)+\operatorname{dim}\left(\varepsilon_{\varrho_{2}}\right)+\operatorname{dim}\left(\varepsilon_{\varrho_{3}}\right)=3 p \\
& =\operatorname{dim}(L)+\operatorname{dim}\left(\mu_{\varrho_{1}}\right)+\operatorname{dim}\left(\mu_{\varrho_{2}}\right)+\operatorname{dim}\left(\mu_{\varrho_{3}}\right) \geq 2 q
\end{aligned}
$$

and $q>p$, one necessarily has $\operatorname{dim}(L)+\operatorname{dim}\left(\mu_{\varrho_{1}}\right)+\operatorname{dim}\left(\mu_{\varrho_{2}}\right)+\operatorname{dim}\left(\mu_{\varrho_{3}}\right)=2 q$ and there is only one $\mu_{\varrho_{i}}$ with $\mu_{\varrho_{i}} \equiv \tau$. By Lemma 3.2, there is then an automorphism $\sigma: Z_{2}^{2} \rightarrow Z_{2}^{2}$ such that $\left(L ;\left\{\mu_{\varrho_{1}}, \mu_{\varrho_{2}}, \mu_{\varrho_{3}}\right\}\right)$ is simultaneously cobordant to the fixed data of the $Z_{2}^{2}$-action $\sigma \Gamma_{1}^{2}(L \times L ; t)$, and hence $\left(F_{K} ; \Phi_{\mid G}\right) \cup \sigma \Gamma_{1}^{2}(L \times L ; t)$ is equivariantly cobordant to a $Z_{2}^{2}$-action with fixed data $\left(K ;\left\{\varepsilon_{\varrho_{1}}, \varepsilon_{\varrho_{2}}, \varepsilon_{\varrho_{3}}\right\}\right)$. This again contradicts Lemma 3.8, which forces $\varepsilon_{\varrho_{3}} \equiv \tau$ and $\Omega$ to be a subgroup of $\operatorname{Hom}\left(Z_{2}^{k}, Z_{2}\right)$. In particular, the number of bundles $\varepsilon_{\varrho}$ with $\varepsilon_{\varrho} \equiv \tau$ is $2^{r}-1$ for some $2 \leq r \leq k$ ( $r$ is the dimension of $\Omega$ as $Z_{2}$-vector space) and $m=2^{r} p$.

By Lemma 3.1, there exist subgroups $G, H \subset Z_{2}^{k}$ with $G$ isomorphic to $Z_{2}^{r}$ and $Z_{2}^{k}=G \oplus H$, so that the fixed data of $G$ acting on the fixed point set $F_{H}$ of $H$ is $\left(K ;\left\{\varepsilon_{\varrho}\right\}_{\varrho \in \Omega \cap \mathcal{P}}\right) \cup\left(L ;\left\{\mu_{\varrho}\right\}_{\varrho \in \Omega \cap \mathcal{P}}\right)$. As in the previous argument, write $F_{H}=F_{K} \cup F_{L}$. Since $\varepsilon_{\varrho}=0$ for every $\varrho \in \mathcal{P}-(\Omega-\{1\})$, $M=F_{K}=F_{L}=F_{H}$. Then one has a $Z_{2}^{r}$-action $\left(M ; \Phi_{\mid G}\right)$ with fixed data 
$\left(K ;\left\{\varepsilon_{\varrho}\right\}_{\varrho \in \Omega \cap \mathcal{P}}\right) \cup\left(L ;\left\{\mu_{\varrho}\right\}_{\varrho \in \Omega \cap \mathcal{P}}\right)$ where, for each $\varrho \in \Omega \cap \mathcal{P} \operatorname{dim}\left(\varepsilon_{\varrho}\right)=$ $\operatorname{dim}(K)$ and either $\mu_{\varrho}=0$ or $\operatorname{dim}\left(\mu_{\varrho}\right)+\operatorname{dim}(L)=2 q>2 p=\operatorname{dim}\left(\varepsilon_{\varrho}\right)+$ $\operatorname{dim}(K)$. By Lemma 3.5, it follows that the list $\left(K ;\left\{\varepsilon_{\varrho}\right\}_{\varrho \in \Omega \cap \mathcal{P}}\right)$ is simultaneously cobordant to $\left(K ;\left\{\tau_{\varrho}\right\}_{\varrho \in \Omega \cap \mathcal{P}}\right)$, where, for each $\varrho \in \Omega \cap \mathcal{P}$, $\tau_{\varrho}$ is the tangent bundle $\tau \rightarrow K$.

Now choose a basis $\left(T_{1}^{\prime}, \ldots, T_{r}^{\prime}, T_{r+1}^{\prime \prime}, \ldots, T_{k}^{\prime \prime}\right)$ for $Z_{2}^{k}$ so that $\left(T_{1}^{\prime}, \ldots, T_{r}^{\prime}\right)$ is a basis for $G$ and $\left(T_{r+1}^{\prime \prime}, \ldots, T_{k}^{\prime \prime}\right)$ is a basis for $H$. Consider the automorphism $\varphi: G \rightarrow G$ where $\varphi\left(T_{i}\right)=T_{i}^{\prime}$ if $1 \leq i \leq r$ and $\varphi\left(T_{i}\right)=T_{i}^{\prime \prime}$ if $r<i \leq k$, and the $Z_{2}^{k}$-action $\varphi\left(M^{m} ; \Phi\right)$. To describe the part of the fixed data of this action over $K$, note that if $\varrho \in \mathcal{P}$ is the trivial homomorphism on $H$, then $\varrho \in \Omega$ and thus $\varepsilon_{\varrho} \equiv \tau$; otherwise, $\varrho \notin \Omega$, which means that $\varepsilon_{\varrho}=0$. Since the list $\left\{\varepsilon_{\varrho}\right\}_{\varrho \in \Omega \cap \mathcal{P}}$ is simultaneously cobordant to $\left\{\tau_{\varrho}\right\}_{\varrho \in \Omega \cap \mathcal{P}}$, the part of the fixed data of $\varphi\left(M^{m} ; \Phi\right)$ over $K$ is then simultaneously cobordant to the list $\left\{\varepsilon_{\varrho}\right\}_{\varrho \in \mathcal{P}}$ given by $\varepsilon_{\varrho}=\tau$ when $\varrho$ is the trivial homomorphism on $H$ and $\varepsilon_{\varrho}=0$ otherwise, which in turn is the fixed data of the $Z_{2}^{k}$-action $\Gamma_{r}^{k}(K \times K ; t)$. Setting $\sigma=\varphi^{-1}$, one sees that the $Z_{2}^{k}$-action $(M ; \Phi) \cup \sigma \Gamma_{r}^{k}(K \times K ; t)$ is equivariantly cobordant to a $Z_{2}^{k}$-action fixing $L$. By Lemma 3.8, this action is equivariantly cobordant to $\sigma^{\prime} \Gamma_{s}^{k}(L \times L ; t)$ for some automorphism $\sigma^{\prime}: Z_{2}^{k} \rightarrow Z_{2}^{k}$ and some $1 \leq s \leq k$, which gives the result.

REMARK. Note that, if the $Z_{2}^{k}$-action $(M ; \Phi)$ is equivariantly cobordant to $\sigma \Gamma_{r}^{k}(K \times K ; t) \cup \sigma^{\prime} \Gamma_{s}^{k}(L \times L ; t)$, then $\operatorname{dim}(M)=2^{r} \operatorname{dim}(K)=2^{s} \operatorname{dim}(L)$. Writing $\operatorname{dim}(K)=2^{a} b$ and $\operatorname{dim}(L)=2^{c} d$, where $b$ and $d$ are odd, one then has $b=d$. Thus, if $b \neq d$, there is no action of the above type fixing $K \cup L$.

In the terminology of Section 1, Theorem 3.9 says that, under the hypotheses in question, $(M ; \Phi)$ belongs to $\mathcal{P}_{k, \tau}^{m}(K \cup L) \subset \mathcal{P}_{k}(K \cup L)$, where $\tau$ is the partition $\{\{K\},\{L\}\}$. Therefore we can assume from now on that there is at least one $\varrho \in \mathcal{P}$ for which $U_{\varrho}=V_{\varrho}$. Writing as before $M=M_{K} \cup M_{L}$, one has in this case $M=M_{K}=M_{L}$ connected because $M_{K} \supset U_{\varrho}=V_{\varrho} \subset M_{L}$. The following result closes the task proposed in this section.

Theorem 3.10. Let $(M ; \Phi)$ be a $Z_{2}^{k}$-action with fixed data $\left(K ;\left\{\varepsilon_{\varrho}\right\}_{\varrho \in \mathcal{P}}\right)$ $\cup\left(L ;\left\{\mu_{\varrho}\right\}_{\varrho \in \mathcal{P}}\right)$ as above. Write $\mathcal{P}_{1}=\left\{\varrho \in \mathcal{P} \mid\left(\varepsilon_{\varrho}, \mu_{\varrho}\right) \equiv(\eta, \eta)\right\}, \mathcal{P}_{2}=$ $\left\{\varrho \in \mathcal{P} \mid\left(\varepsilon_{\varrho}, \mu_{\varrho}\right) \equiv(\tau, \tau)\right\}$ and $\mathcal{P}_{3}=\left\{\varrho \in \mathcal{P} \mid\left(\varepsilon_{\varrho}, \mu_{\varrho}\right)=(0,0)\right\}$. Then $\mathcal{P}=\mathcal{P}_{1} \cup \mathcal{P}_{2} \cup \mathcal{P}_{3}, \mathcal{P}_{1}$ has $2^{r-1}$ elements and $\mathcal{P}_{2}$ has $2^{r-1}-1$ elements for some $1 \leq r \leq k$ (consequently, $\mathcal{P}_{3}$ has $2^{k}-2^{r}$ elements). Further, there is an automorphism $\sigma: Z_{2}^{k} \rightarrow Z_{2}^{k}$ such that the pairs $\left(\varepsilon_{\varrho}, \mu_{\varrho}\right)$ are indexed by $\mathcal{P}$ following the pattern of an action of the type $\sigma \Gamma_{r}^{k}(W ; T)$, where $(W ; T)$ is an involution fixing $K \cup L$ with $W$ connected.

Proof. By hypothesis, there is at least one $\varrho_{0} \in \mathcal{P}$ for which $U_{\varrho_{0}}=V_{\varrho_{0}}$, and $\left(\varepsilon_{\varrho_{0}}, \mu_{\varrho_{0}}\right) \equiv(\eta, \eta)$. For any $\varrho \in \mathcal{P}-\left\{\varrho_{0}\right\}$, we apply Lemma 3.1 to 
the subgroup $\left\{1, \varrho_{0}, \varrho, \varrho_{0} \varrho\right\} \subset \operatorname{Hom}\left(Z_{2}^{k}, Z_{2}\right)$ to conclude that there exist subgroups $G, H \subset Z_{2}^{k}$ with $G$ isomorphic to $Z_{2}^{2}$ and $Z_{2}^{k}=G \oplus H$, so that the fixed data of the $Z_{2}^{2}$-action obtained by letting $G$ act on the fixed point set $F_{H}$ of $H$ is $\left(K ;\left\{\varepsilon_{\varrho_{0}}, \varepsilon_{\varrho}, \varepsilon_{\varrho \varrho \varrho}\right\}\right) \cup\left(L ;\left\{\mu_{\varrho_{0}}, \mu_{\varrho}, \mu_{\varrho \varrho \varrho}\right\}\right)$. By the argument outlined before Lemma 3.1, $H \subset \operatorname{ker}\left(\varrho_{0}\right)$; writing as before $F_{H}=F_{K} \cup F_{L}$, one then has $U_{\varrho_{0}}=V_{\varrho_{0}} \subset F_{H}$, and thus $F_{H}=F_{K}=F_{L}$ is connected. By Lemma 3.7, one has the following possibilities:

(i) $\left(\varepsilon_{\varrho}, \mu_{\varrho}\right)=(0,0)$ and $\left(\varepsilon_{\varrho_{\varrho} \varrho}, \mu_{\varrho} \varrho\right)=(0,0)$;

(ii) $\left(\varepsilon_{\varrho}, \mu_{\varrho}\right)=(\eta, \eta)$ and $\left(\varepsilon_{\varrho_{0} \varrho}, \mu_{\varrho_{0} \varrho}\right)=(\tau, \tau)$; or

(iii) $\left(\varepsilon_{\varrho}, \mu_{\varrho}\right)=(\tau, \tau)$ and $\left(\varepsilon_{\varrho_{0} \varrho}, \mu_{\varrho_{0}}\right)=(\eta, \eta)$.

This gives $\mathcal{P}=\mathcal{P}_{1} \cup \mathcal{P}_{2} \cup \mathcal{P}_{3}$; further, this shows that $\varrho \mapsto \varrho_{0} \varrho$ determines a bijection between $\mathcal{P}_{1}$ and $\mathcal{P}_{2} \cup\{1\}$, with inverse given by the same rule.

We assert that $\mathcal{P}_{2} \cup\{1\}$ is a subgroup of $\operatorname{Hom}\left(Z_{2}^{k}, Z_{2}\right)$. In fact, take $\varrho_{1}, \varrho_{2} \in \mathcal{P}_{2}$ with $\varrho_{1} \neq \varrho_{2}$. As before, Lemma 3.1 gives a $Z_{2}^{2}$-action $\left(F_{K} \cup F_{L} ; \psi\right)$ with fixed data $\left(K ;\left\{\varepsilon_{\varrho_{1}}, \varepsilon_{\varrho_{2}}, \varepsilon_{\varrho_{1} \varrho_{2}}\right\}\right) \cup\left(L ;\left\{\mu_{\varrho_{1}}, \mu_{\varrho_{2}}, \mu_{\varrho_{1} \varrho_{2}}\right\}\right)$. Suppose $F_{K}=F_{L}$, and set $x=\operatorname{dim}\left(\varepsilon_{\varrho_{1} \varrho_{2}}\right)$, and $y=\operatorname{dim}\left(\mu_{\varrho_{1} \varrho_{2}}\right)$. If $\left(\varepsilon_{\varrho_{1} \varrho_{2}}, \mu_{\varrho_{1} \varrho_{2}}\right)$ $\equiv(\eta, \eta)$, then the $Z_{2}^{2}$-action $\left(F_{K} \cup F_{L} ; \psi\right)$ contradicts Lemma 3.7 because $(\tau, \tau) \neq(\eta, \eta)$. Since $3 p+x=3 q+y$ and $p<q$, we find that $x>y$ and thus $x \neq 0$. This means that we cannot have $\left(\varepsilon_{\varrho_{1} \varrho_{2}}, \mu_{\varrho_{1} \varrho_{2}}\right) \equiv(\tau, \tau),(0, \tau)$ or $(0,0)$, and there remains the possibility $\left(\varepsilon_{\varrho_{1} \varrho_{2}}, \mu_{\varrho_{1} \varrho_{2}}\right) \equiv(\tau, 0)$. In this case, $\left(F_{K} \cup F_{L} ; \psi\right)$ satisfies the hypotheses of Lemma 3.5 , and thus $\left(\varepsilon_{\varrho_{1}}, \varepsilon_{\varrho_{2}}, \varepsilon_{\varrho_{1} \varrho_{2}}\right)$ is simultaneously cobordant to $\left(\tau_{K}, \tau_{K}, \tau_{K}\right)$, where $\tau_{K} \rightarrow K$ is the tangent bundle of $K$. It follows that the $Z_{2}^{2}$-action $\left(F_{K} \cup F_{L} ; \psi\right) \cup \Gamma_{2}^{2}(K \times K ; t)$ is equivariantly cobordant to a $Z_{2}^{2}$-action with fixed data $\left(L ;\left\{\mu_{\varrho_{1}}, \mu_{\varrho_{2}}, \mu_{\varrho_{1} \varrho_{2}}\right\}\right)$, which contradicts Lemma 3.8. Hence $F_{K} \cap F_{L}=\emptyset$, and we can apply Lemma 3.8 to the $Z_{2}^{2}$-actions $\left(F_{K} ; \psi\right)$ and $\left(F_{L} ; \psi\right)$ to conclude that $\left(\varepsilon_{\varrho_{1} \varrho_{2}}, \mu_{\varrho_{1} \varrho_{2}}\right) \equiv$ $(\tau, \tau)$, thus showing the assertion. It follows that $\mathcal{P}_{2}$ has $2^{r-1}-1$ elements, and consequently $\mathcal{P}_{1}$ has $2^{r-1}$ elements for some $2 \leq r \leq k$.

To prove the last statement of the theorem, first note that $\mathcal{P}_{1} \cup \mathcal{P}_{2} \cup\{1\}$ is a subgroup of $\operatorname{Hom}\left(Z_{2}^{k}, Z_{2}\right)$ isomorphic to $Z_{2}^{r}$ : if $\varrho_{1}, \varrho_{2} \in \mathcal{P}_{1}$, then $\varrho_{0} \varrho_{1}$ and $\varrho_{0} \varrho_{2}$ belong to the subgroup $\mathcal{P}_{2} \cup\{1\}$ and $\left(\varrho_{0} \varrho_{1}\right)\left(\varrho_{0} \varrho_{2}\right)=\varrho_{1} \varrho_{2} \in \mathcal{P}_{2} \cup\{1\}$. If $\varrho_{1} \in \mathcal{P}_{1}$ and $\varrho_{2} \in \mathcal{P}_{2}$, then $\varrho_{2}$ can be written as $\varrho_{2}=\varrho_{1}^{\prime} \varrho_{0}$ with $\varrho_{1}^{\prime} \in \mathcal{P}_{1}$, and $\varrho_{1} \varrho_{1}^{\prime} \in \mathcal{P}_{2} \cup\{1\}$ by the previous argument; thus $\varrho_{1} \varrho_{2}=$ $\varrho_{0}\left(\varrho_{1} \varrho_{1}^{\prime}\right) \in \mathcal{P}_{1}$. In this way, Lemma 3.1 gives a decomposition $Z_{2}^{k}=G \oplus H$, with $G$ isomorphic to $Z_{2}^{r}$ and with the $Z_{2}^{r}$-action $\left(F_{H} ; \Phi_{\mid G}\right)$ having fixed data $\left(K ;\left\{\varepsilon_{\varrho}\right\}_{\varrho \in \mathcal{P}_{1} \cup \mathcal{P}_{2}}\right) \cup\left(L ;\left\{\mu_{\varrho}\right\}_{\varrho \in \mathcal{P}_{1} \cup \mathcal{P}_{2}}\right)$. For dimensional reasons, $F_{H}=$ $F_{K} \cup F_{L}=F_{K}=F_{L}=M$, and thus each element of $H$ acts in $M$ as the identity involution. Applying again Lemma 3.1 to the action $\left(M ; \Phi_{\mid G}\right)$ and the subgroup $\mathcal{P}_{2} \cup\{1\} \subset \mathcal{P}_{1} \cup \mathcal{P}_{2} \cup\{1\}$, one obtains a decomposition $G=G_{1} \oplus G_{2}$ and the action $\left(F_{G_{2}} ; \Phi_{\mid G_{1}}\right)$ with fixed data $\left(K ;\left\{\varepsilon_{\varrho}\right\}_{\varrho \in \mathcal{P}_{2}}\right) \cup\left(L ;\left\{\mu_{\varrho}\right\}_{\varrho \in \mathcal{P}_{2}}\right)$. Since $G_{1}$ is isomorphic to $Z_{2}^{r-1}, G_{2}$ is isomorphic to $Z_{2}$; denote by $S$ the 
generator of $G_{2}$ and by $\nu \rightarrow F_{G_{2}}=F_{S}$ the normal bundle of $F_{G_{2}}$ in $M$. One knows that $S$ acts as -1 in $\nu$. Since

$$
\nu_{\mid K \cup L}=\left(\bigoplus_{\varrho \in \mathcal{P}_{1}} \varepsilon_{\varrho} \rightarrow K\right) \cup\left(\bigoplus_{\varrho \in \mathcal{P}_{1}} \mu_{\varrho} \rightarrow L\right),
$$

$S$ acts as -1 in $\varepsilon_{\varrho}$ and in $\mu_{\varrho}$ for every $\varrho \in \mathcal{P}_{1}$. Evidently, $S$ acts as 1 in $F_{G_{2}}=F_{S}$ and thus $S$ acts as 1 in $\varepsilon_{\varrho}$ and in $\mu_{\varrho}$ for every $\varrho \in \mathcal{P}_{2}$. Choose $\tau_{r+1}, \ldots, \tau_{k}$ generating $H$ (one may have $H=\{1\}$, in which case this step is unnecessary) and $f_{2}, \ldots, f_{r}$ generating $G_{1}$. Consider the automorphism $\varphi: Z_{2}^{k} \rightarrow Z_{2}^{k}$ given by $\varphi\left(T_{1}\right)=S, \varphi\left(T_{i}\right)=f_{i}$ if $2 \leq i \leq r$ and $\varphi\left(T_{i}\right)=\tau_{i}$ if $r+1 \leq i \leq k$. Then in the fixed data of the $Z_{2}^{k}$-action $\varphi(M ; \Phi)$ the pairs $\left(\varepsilon_{\varrho}, \mu_{\varrho}\right)$ are indexed by $\mathcal{P}$ following the pattern of an action of the type $\Gamma_{r}^{k}(W ; T)$, where $(W ; T)$ is an involution fixing $K \cup L$ with $W$ connected. Setting $\sigma=\varphi^{-1}$, one gets the result.

4. Simultaneous cobordism of the fixed data. As we have seen, a $Z_{2}^{k}$-action $(M ; \Phi)$ as in Theorem 3.10 gives rise to a set of $2^{r-1}$ involutions $\left(U_{\varrho} \cup V_{\varrho} ; T_{\varrho}\right), \varrho \in \mathcal{P}_{1}$, where $\operatorname{dim}\left(U_{\varrho}\right)=\operatorname{dim}\left(V_{\varrho}\right), T_{\varrho} \notin \operatorname{ker}(\varrho)$ and the fixed point set of $T_{\varrho}$ is $K \cup L$. These involutions determine, in turn, a subset of the set of all equivariant cobordism classes of involutions fixing $K \cup L$, $\mathcal{A}_{1}(K \cup L)$. In order to better understand this subset, one needs to know some additional facts about it. In general, for a given $F$ which is not a boundary, $\mathcal{A}_{1}(F)$ may be empty (take for instance $F=S^{n} \cup\{$ point $\}, S^{n}$ the $n$-sphere with $n \neq 1,2,4$ and 8 [2, Theorem 27.6]) and it is always finite (this follows from the strengthened Boardman $5 / 2$-theorem of [3]). Denote by $R^{j}$ (resp. by $R$ ) the trivial $j$-dimensional resp. (resp. one-dimensional) vector bundle over any base space. An element $\left[\left(W^{n} ; T\right)\right] \in \mathcal{A}_{1}(F)$ is determined by the cobordism class of the normal bundle $\eta \rightarrow F$, which is a union of bundles over the components of $F$. There is a greatest natural number $p \geq 0$ for which $\eta$ is cobordant to a bundle $\kappa \oplus R^{p} \rightarrow F$ ( $p$ is the same over all the components). Now one knows from [2, Theorem 26.4] that if $\eta \rightarrow F$ is the fixed data of an involution and $\eta \rightarrow F$ is equivalent to $\eta^{\prime} \oplus R \rightarrow F$, then $\eta^{\prime} \rightarrow F$ is also the fixed data of an involution. It follows that there are involutions $\left(\bar{W}^{n-p+i} ; T\right)$ fixing $F$ for which the normal bundle of $F$ in $\bar{W}^{n-p+i}$ is $\kappa \oplus R^{i}$ for $0 \leq i \leq p$, with $\left(\bar{W}^{n-p+p} ; T\right)$ cobordant to $\left(W^{n} ; T\right)$. Further, one knows how to add additional trivial bundles to the normal bundle of an involution. One may form

$$
\Gamma\left(W^{n} ; T\right)=\left(\frac{S^{1} \times W^{n}}{-1 \times T} ; \text { conjugation } \times 1\right) .
$$

The fixed point set of this involution consists of a copy of $F$ with normal bundle $\eta \oplus R \rightarrow F$ and a copy of $W^{n}$ with normal bundle $R \rightarrow W^{n}$. If $W^{n}$ 
bounds as a manifold, then $R \rightarrow W^{n}$ bounds as a bundle and $\Gamma\left(W^{n} ; T\right)$ is cobordant to an involution with fixed data $\eta \oplus R \rightarrow F$. This procedure of removing sections and adding trivial bundles provides a subset of $\mathcal{A}_{1}(F)$ with representatives having fixed data $\kappa \oplus R^{i} \rightarrow F, 0 \leq i \leq p+t, t \geq 0$, where, in the process of adding trivial bundles, $t$ is the first number for which the involution $\left(\bar{W}^{n+t} ; T\right)$ has the underlying manifold $\bar{W}^{n+t}$ nonbounding, and $\mathcal{A}_{1}(F)$ is a disjoint union of subsets of this type.

Now, if $(W ; T)$ has fixed data $\eta \rightarrow F$, then the fixed data of a $Z_{2}^{k}$-action obtained by removing sections from the fixed data of an action of the type $\sigma \Gamma_{r}^{k}(W ; T)$ has $2^{r-1}$ eigenbundles obtained by removing sections from $\eta$. Thus, the corresponding $2^{r-1}$ involutions (fixing $F$ ) belong to the same subset of $\mathcal{A}_{1}(F)$. Also this fixed data has $2^{r-1}-1$ eigenbundles obtained by removing sections from the tangent bundle of $F$; in particular, if some component of $F$ has property $\mathcal{H}$, then the tangent bundle over this component has no sections (see [8]), and thus no section can be removed from these $2^{r-1}-1$ eigenbundles over $F$. By comparison, our next (and final) task is to show that the $2^{r-1}$ involutions $\left(U_{\varrho} \cup V_{\varrho} ; T_{\varrho}\right), \varrho \in \mathcal{P}_{1}$, given by Theorem 3.10, belong to the same subset of $\mathcal{A}_{1}(K \cup L)$, and that the lists $\left(K ;\left\{\varepsilon_{\varrho}\right\}_{\varrho \in \mathcal{P}}\right)$ and $\left(L ;\left\{\mu_{\varrho}\right\}_{\varrho \in \mathcal{P}}\right)$, with the description in terms of individual cobordism given by Theorem 3.10, are simultaneously cobordant to lists over $K$ and $L$ respectively, as described above.

This will be done by combining Lemma 3.4 with the following

LEMma 4.1. Let $F$ be a connected closed manifold, $\varepsilon \rightarrow F$ a vector bundle over $F$ with $\operatorname{dim}(\varepsilon)=\operatorname{dim}(F)$ and $\xi \rightarrow R P(\varepsilon)$ the usual line bundle. Suppose that $\left\{\kappa_{\varrho}\right\}$ and $\left\{\kappa_{\varrho}^{\prime}\right\}$ are lists of vector bundles over $F$, indexed by the same set $\mathcal{Q}$, such that the list $\left(R P(\varepsilon) ; \xi,\left\{\kappa_{\varrho} \oplus\left(\xi \otimes \kappa_{\varrho}^{\prime}\right)\right\}_{\varrho \in \mathcal{Q}}\right)$ is a (simultaneous) boundary. Choose any $\varrho_{0} \in \mathcal{Q}$, and set $p=\operatorname{dim}\left(\kappa_{\varrho_{0}}\right), q=\operatorname{dim}\left(\kappa_{\varrho_{0}}^{\prime}\right)$. Then the list $\left(F ; \varepsilon, \kappa_{\varrho_{0}}, \kappa_{\varrho_{0}}^{\prime},\left\{\kappa_{\varrho}, \kappa_{\varrho}^{\prime}\right\}_{\varrho \in \mathcal{Q}-\left\{\varrho_{0}\right\}}\right)$ is simultaneously cobordant

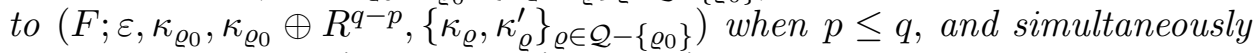
cobordant to $\left(F ; \varepsilon, \kappa_{\varrho_{0}}^{\prime} \oplus R^{p-q}, \kappa_{\varrho_{0}}^{\prime},\left\{\kappa_{\varrho}, \kappa_{\varrho}^{\prime}\right\}_{\varrho \in \mathcal{Q}-\left\{\varrho_{0}\right\}}\right)$ when $p \geq q$.

Proof. Set $n=\operatorname{dim}(F)=\operatorname{dim}(\varepsilon), m_{\varrho}=\operatorname{dim}\left(\kappa_{\varrho}\right)$ and $n_{\varrho}=\operatorname{dim}\left(\kappa_{\varrho}^{\prime}\right)$ for each $\varrho \in \mathcal{Q}$; in particular, $m_{\varrho 0}=p$ and $n_{\varrho_{0}}=q$. Let

$$
\begin{aligned}
W(F) & =1+w_{1}+\cdots+w_{n}, \\
W(\varepsilon) & =1+\theta_{1}+\cdots+\theta_{n}, \\
W\left(\kappa_{\varrho}\right) & =1+u_{1}^{\varrho} \cdots+u_{m_{\varrho}}^{\varrho}, \\
W\left(\kappa_{\varrho}^{\prime}\right) & =1+v_{1}^{\varrho}+\cdots+v_{n_{\varrho}}^{\varrho}
\end{aligned}
$$

be the respective Stiefel-Whitney classes. Letting $c \in H^{1}\left(R P(\varepsilon) ; Z_{2}\right)$ be the first Stiefel-Whitney class of the line bundle $\xi$, one knows that the 
Stiefel-Whitney class of $R P(\varepsilon)$ is $W(R P(\varepsilon))=\left(1+w_{1}+\cdots+w_{n}\right) \cdot\left\{(1+c)^{n}+\theta_{1}(1+c)^{n-1}+\cdots+\theta_{n-1}(1+c)+\theta_{n}\right\}$, the Stiefel-Whitney class of $\xi$ is $W(\xi)=1+c$, and the Stiefel-Whitney class of $\kappa_{\varrho} \oplus\left(\xi \otimes \kappa_{\varrho^{\prime}}\right)$ is $W\left(\kappa_{\varrho} \oplus\left(\xi \otimes \kappa_{\varrho^{\prime}}\right)\right)=\left(1+u_{1}^{\varrho}+\cdots+u_{m_{\varrho}}^{\varrho}\right)$ $\cdot\left\{(1+c)^{n_{\varrho}}+v_{1}^{\varrho}(1+c)^{n_{\varrho}-1}+\cdots+v_{n_{\varrho}-1}^{\varrho}(1+c)+v_{n_{\varrho}}^{\varrho}\right\}$.

Because $\left(R P(\varepsilon) ; \xi,\left\{\kappa_{\varrho} \oplus\left(\xi \otimes \kappa_{\varrho}^{\prime}\right)\right\}_{\varrho \in \mathcal{Q}}\right)$ is a boundary, any class of dimension $2 n-1$ given by a product of classes $w_{i}(R P(\varepsilon)), c$ and $w_{j}\left(\kappa_{\varrho} \oplus\left(\xi \otimes \kappa_{\varrho^{\prime}}\right)\right)$ gives a zero characteristic number for $R P(\varepsilon)$. We will apply this using certain special classes, which are polynomials in the above classes, and were introduced in [9]. Specifically, for any $r$, one lets

That is,

$$
W[r]=\frac{W(R P(\varepsilon))}{(1+c)^{n-r}} \quad \text { and } \quad W_{\varrho}[r]=\frac{W\left(\kappa_{\varrho} \oplus\left(\xi \otimes \kappa_{\varrho^{\prime}}\right)\right)}{(1+c)^{n_{\varrho}-r}} .
$$

$$
\begin{aligned}
W[r]= & \left(1+w_{1}+\cdots+w_{n}\right) \\
& \cdot\left\{(1+c)^{r}+\theta_{1}(1+c)^{r-1}+\cdots+\theta_{r}+\frac{\theta_{r+1}}{1+c}+\cdots+\frac{\theta_{n}}{(1+c)^{n-r}}\right\}, \\
W_{\varrho}[r]= & \left(1+u_{1}^{\varrho}+\cdots+u_{m_{\varrho}}^{\varrho}\right) \\
& \cdot\left\{(1+c)^{r}+v_{1}^{\varrho}(1+c)^{r-1}+\cdots+v_{r}^{\varrho}+\frac{v_{r+1}^{\varrho}}{1+c}+\cdots+\frac{v_{n_{\varrho}}^{\varrho}}{(1+c)^{n_{\varrho}-r}}\right\} .
\end{aligned}
$$

These classes have the following special properties (see [9]):

$W[r]_{2 r}=w_{r} c^{r}+$ terms with smaller $c$ powers,

$W[r]_{2 r+1}=\left(w_{r+1}+\theta_{r+1}\right) c^{r}+$ terms with smaller $c$ powers,

$W[r]_{2 r+2}=\theta_{r+1} c^{r+1}+$ terms with smaller $c$ powers,

and in the same way,

$W_{\varrho}[r]_{2 r}=u_{r}^{\varrho} c^{r}+$ terms with smaller $c$ powers,

$W_{\varrho}[r]_{2 r+1}=\left(u_{r+1}^{\varrho}+v_{r+1}^{\varrho}\right) c^{r}+$ terms with smaller $c$ powers,

$W_{\varrho}[r]_{2 r+2}=v_{r+1}^{\varrho} c^{r+1}+$ terms with smaller $c$ powers.

For a sequence $\omega=\left(i_{1}, \ldots, i_{s}\right)$ of natural numbers, one lets $|\omega|=i_{1}+$ $\cdots+i_{s}$, and for $w=1+w_{1}+\cdots+w_{p}$, one lets $w_{\omega}=w_{i_{1}} \cdots w_{i_{s}}$ be the product of the classes $w_{i}$.

Then given sequences $\omega=\left(i_{1}, \ldots, i_{s}\right), \omega^{\prime}=\left(j_{1}, \ldots, j_{t}\right), \omega_{\varrho}=\left(i_{1}^{\varrho}, \ldots, i_{s_{\varrho}}^{\varrho}\right)$ and $\omega_{\varrho}^{\prime}=\left(j_{1}^{\varrho}, \ldots, j_{t_{\varrho}}^{\varrho}\right)$ for each $\varrho \in \mathcal{Q}$, and a natural number $1 \leq r \leq$ $\max \{p, q\}$ with

$$
|\omega|+\left|\omega^{\prime}\right|+\sum_{\varrho}\left|\omega_{\varrho}\right|+\sum_{\varrho}\left|\omega_{\varrho}^{\prime}\right|+r=n
$$


one may form the class

$$
\begin{aligned}
X= & \left(\prod_{i \in \omega} W[i]_{2 i}\right) \cdot\left(\prod_{i \in \omega^{\prime}} W[i-1]_{2 i}\right) \\
& \cdot \prod_{\varrho \neq \varrho_{0}}\left\{\left(\prod_{i \in \omega_{\varrho}} W_{\varrho}[i]_{2 i}\right) \cdot\left(\prod_{i \in \omega_{\varrho}^{\prime}} W_{\varrho}[i-1]_{2 i}\right)\right\} \\
& \cdot\left(\prod_{i \in \omega_{\varrho}} W_{\varrho_{0}}[i]_{2 i}\right) \cdot\left(\prod_{i \in \omega_{\varrho_{0}}^{\prime}} W_{\varrho_{0}}[i-1]_{2 i}\right) \cdot W_{\varrho_{0}}[r-1]_{2 r-1} .
\end{aligned}
$$

Since $X$ has dimension $2 n-1$ and is a polynomial in the characteristic classes of $R P(\varepsilon), \xi$ and $\kappa_{\varrho} \oplus\left(\xi \otimes \kappa_{\varrho^{\prime}}\right)$, it gives the zero characteristic number $X[R P(\varepsilon)]$ for $R P(\varepsilon)$. From the properties listed above, one has

$$
\begin{gathered}
\prod_{i \in \omega} W[i]_{2 i}=W(F)_{\omega} \cdot c^{|\omega|}+\text { terms with smaller } c \text { powers, } \\
\prod_{i \in \omega^{\prime}} W[i-1]_{2 i}=W(\varepsilon)_{\omega^{\prime}} \cdot c^{\left|\omega^{\prime}\right|}+\text { terms with smaller } c \text { powers, } \\
\prod_{i \in \omega_{\varrho}} W_{\varrho}[i]_{2 i}=W\left(\kappa_{\varrho}\right)_{\omega_{\varrho}} \cdot c^{\left|\omega_{\varrho}\right|}+\text { terms with smaller } c \text { powers, } \\
\prod_{i \in \omega_{\varrho}^{\prime}} W_{\varrho}[i-1]_{2 i}=W\left(\kappa_{\varrho}^{\prime}\right)_{\omega_{\varrho}^{\prime}} \cdot c^{\left|\omega_{\varrho}^{\prime}\right|}+\text { terms with smaller } c \text { powers, } \\
W_{\varrho}[r-1]_{2 r-1}=\left(u_{r}^{\varrho_{0}}+v_{r}^{\varrho_{0}}\right) \cdot c^{r}+\text { terms with smaller } c \text { powers. }
\end{gathered}
$$

It follows that

$$
\begin{aligned}
X= & W(F)_{\omega} \cdot W(\varepsilon)_{\omega^{\prime}} \cdot \prod_{\varrho \neq \varrho_{0}}\left(W\left(\kappa_{\varrho}\right)_{\omega_{\varrho}} \cdot W\left(\kappa_{\varrho}^{\prime}\right)_{\omega_{\varrho}^{\prime}}\right) \cdot W\left(\kappa_{\varrho_{0}}\right)_{\omega_{\varrho_{0}}} \cdot W\left(\kappa_{\varrho_{0}}^{\prime}\right)_{\omega_{\varrho_{0}}^{\prime}} \\
& \cdot\left(u_{r}^{\varrho_{0}}+v_{r}^{\varrho_{0}}\right) \cdot c^{n-1}+\text { terms with smaller } c \text { powers. }
\end{aligned}
$$

Now if a term of dimension $2 n-1$ involves a power of $c$ less than $n-1$, it necessarily has a factor of dimension greater than $n$ coming from the cohomology of $F$, which is zero. Also one knows that $H^{*}\left(R P(\varepsilon) ; Z_{2}\right)$ is the free $H^{*}\left(F ; Z_{2}\right)$-module on $1, c, c^{2}, \ldots, c^{n-1}$. Therefore

$$
\begin{aligned}
0=X[R P(\varepsilon)]= & W(F)_{\omega} \cdot W(\varepsilon)_{\omega^{\prime}} \cdot \prod_{\varrho \neq \varrho_{0}}\left(W\left(\kappa_{\varrho}\right)_{\omega_{\varrho}} \cdot W\left(\kappa_{\varrho}^{\prime}\right)_{\omega_{\varrho}^{\prime}}\right) \\
& \cdot W\left(\kappa_{\varrho_{0}}\right)_{\omega_{\varrho_{0}}} \cdot W\left(\kappa_{\varrho_{0}}^{\prime}\right)_{\omega_{\varrho_{0}}^{\prime}} \cdot\left(u_{r}^{\varrho_{0}}+v_{r}^{\varrho_{0}}\right) \cdot c^{n-1}[R P(\varepsilon)] \\
= & W(F)_{\omega} \cdot W(\varepsilon)_{\omega^{\prime}} \cdot \prod_{\varrho \neq \varrho_{0}}\left(W\left(\kappa_{\varrho}\right)_{\omega_{\varrho}} \cdot W\left(\kappa_{\varrho}^{\prime}\right)_{\omega_{\varrho}^{\prime}}\right) \\
& \cdot W\left(\kappa_{\varrho_{0}}\right)_{\omega_{\varrho_{0}}} \cdot W\left(\kappa_{\varrho_{0}}^{\prime}\right)_{\omega_{\varrho_{0}}^{\prime}} \cdot\left(u_{r}^{\varrho_{0}}+v_{r}^{\varrho_{0}}\right)[F]
\end{aligned}
$$


and thus

$$
\begin{aligned}
& W(F)_{\omega} \cdot W(\varepsilon)_{\omega^{\prime}} \cdot \prod_{\varrho \neq \varrho_{0}}\left(W\left(\kappa_{\varrho}\right)_{\omega_{\varrho}} \cdot W\left(\kappa_{\varrho}^{\prime}\right)_{\omega_{\varrho}^{\prime}}\right) \cdot W\left(\kappa_{\varrho_{0}}\right)_{\omega_{\varrho_{0}}} \cdot W\left(\kappa_{\varrho_{0}}^{\prime}\right)_{\omega_{\varrho_{0}}^{\prime}} \cdot u_{r}^{\varrho_{0}}[F] \\
& =W(F)_{\omega} \cdot W(\varepsilon)_{\omega^{\prime}} \cdot \prod_{\varrho \neq \varrho_{0}}\left(W\left(\kappa_{\varrho}\right)_{\omega_{\varrho}} \cdot W\left(\kappa_{\varrho}^{\prime}\right)_{\omega_{\varrho}^{\prime}}\right) \cdot W\left(\kappa_{\varrho_{0}}\right)_{\omega_{\varrho_{0}}} \cdot W\left(\kappa_{\varrho_{0}}^{\prime}\right)_{\omega_{\varrho_{0}}^{\prime}} \cdot v_{r}^{\varrho_{0}}[F] .
\end{aligned}
$$

This says that any class $u_{r}^{\varrho_{0}}$ and any class $v_{r}^{\varrho_{0}}$ in a characteristic number of $\left(F ; \varepsilon, \kappa_{\varrho_{0}}, \kappa_{\varrho_{0}}^{\prime},\left\{\kappa_{\varrho}, \kappa_{\varrho}^{\prime}\right\}_{\varrho \in \mathcal{Q}-\left\{\varrho_{0}\right\}}\right)$ can be replaced by $v_{r}^{\varrho_{0}}$ and $u_{r}^{\varrho_{0}}$ respectively without changing the value of the characteristic number. In particular, if $p \leq q$ and $p<r \leq q$, then any class $v_{r}^{\rho_{0}}$ can be replaced by the

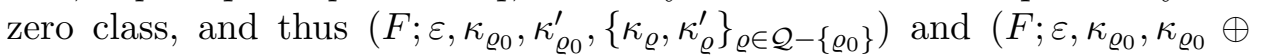
$\left.R^{q-p},\left\{\kappa_{\varrho}, \kappa_{\varrho}^{\prime}\right\}_{\varrho \in \mathcal{Q}-\left\{\varrho_{0}\right\}}\right)$ have the same characteristic numbers. Similarly, $\left(F ; \varepsilon, \kappa_{\varrho_{0}}, \kappa_{\varrho_{0}}^{\prime},\left\{\kappa_{\varrho}, \kappa_{\varrho}^{\prime}\right\}_{\varrho \in \mathcal{Q}-\left\{\varrho_{0}\right\}}\right)$ and $\left(F ; \varepsilon, \kappa_{\varrho_{0}}^{\prime} \oplus R^{p-q}, \kappa_{\varrho_{0}}^{\prime},\left\{\kappa_{\varrho}, \kappa_{\varrho}^{\prime}\right\}_{\varrho \in \mathcal{Q}-\left\{\varrho_{0}\right\}}\right)$ have the same characteristic numbers when $p \geq q$, and the result follows.

Theorem 4.2. Let $(M ; \Phi)$ be a $Z_{2}^{k}$-action as in Theorem 3.10. Let $1 \leq r \leq k$ and $\sigma: Z_{2}^{k} \rightarrow Z_{2}^{k}$ be the automorphism given by Theorem 3.10. Then there exists an involution $(W ; T)$ fixing $K \cup L$ with $W$ connected such that $(M ; \Phi)$ is equivariantly cobordant to a $Z_{2}^{k}$-action obtained by removing sections from the fixed data of $\sigma \Gamma_{r}^{k}(W ; T)$. In other words, $(M ; \Phi)$ belongs to $\mathcal{B}_{k}^{m}(K \cup L) \subset \mathcal{P}_{k}(K \cup L)$.

Proof. The proof is a continuation of the proof of Theorem 3.10. For each $\varrho \in \mathcal{P}_{2}$, we have $U_{\varrho} \cap V_{\varrho}=\emptyset$. Then we can iteratively apply Lemmas 3.3 and 3.4 on the components $U_{\varrho}$ and $V_{\varrho}, \varrho \in \mathcal{P}_{2}$, to conclude that the lists $\left(K ;\left\{\varepsilon_{\varrho}\right\}_{\varrho \in \mathcal{P}}\right.$ and $\left.L ;\left\{\mu_{\varrho}\right\}_{\varrho \in \mathcal{P}}\right)$ are simultaneously cobordant to $\left(K ;\left\{\varepsilon_{\varrho}\right\}_{\varrho \in \mathcal{P}_{1} \cup \mathcal{P}_{3}},\left\{\tau_{\varrho}^{K}\right\}_{\varrho \in \mathcal{P}_{2}}\right)$ and $\left(L ;\left\{\mu_{\varrho}\right\}_{\varrho \in \mathcal{P}_{1} \cup \mathcal{P}_{3}},\left\{\tau_{\varrho}^{L}\right\}_{\varrho \in \mathcal{P}_{2}}\right)$ respectively, where each $\tau_{\varrho}^{K}, \varrho \in \mathcal{P}_{2}$, is the tangent bundle of $K$, and each $\tau_{\varrho}^{L}, \varrho \in \mathcal{P}_{2}$, is the tangent bundle of $L$.

For each $\varrho \in \mathcal{P}_{1}$, set $n_{\varrho}=\operatorname{dim}\left(\varepsilon_{\varrho}\right)$ and $m_{\varrho}=\operatorname{dim}\left(\mu_{\varrho}\right)$. Choose $\varrho_{0} \in \mathcal{P}_{1}$ so that $n_{\varrho_{0}} \leq n_{\varrho}$ for any $\varrho \in \mathcal{P}_{1}$; since $p+n_{\varrho}=q+m_{\varrho}$ when $\varrho \in \mathcal{P}_{1}$, also $m_{\varrho} \leq m_{\varrho}$ for any $\varrho \in \mathcal{P}_{1}$. Taking any $\varrho \in \mathcal{P}_{1}$ with $\varrho \neq \varrho_{0}$, one knows that $\bar{\varrho}=\varrho_{0} \in \mathcal{P}_{2}$ (see the proof of Theorem 3.10), which implies that $U_{\bar{\varrho}} \cap V_{\bar{\varrho}}=\emptyset$. Note that, if $H=\operatorname{ker}(\bar{\varrho})$, then $\varrho_{\mid H}=\varrho_{0 \mid H}$, and if $T \notin H$, then either $\varrho(T)=1$ and $\varrho_{0}(T)=-1$, or $\varrho(T)=-1$ and $\varrho_{0}(T)=1$. This means that, in the argument outlined before Lemma 3.3, $\varrho$ and $\varrho_{0}$ are paired with respect to $\bar{\varrho}$; that is, with the notation of Lemma 3.3, $\left\{\varrho, \varrho_{0}\right\}=\left\{\theta, \theta^{\prime}\right\}$ for some $\theta$.

Then we can iteratively apply Lemmas 3.3 and 4.1 on the (disjoint) components $U_{\bar{\varrho}}$ and $V_{\bar{\varrho}}$, where, for each $\varrho \in \mathcal{P}_{1}-\left\{\varrho_{0}\right\}, \bar{\varrho}=\varrho_{0}$, to conclude that the list $\left(K ;\left\{\varepsilon_{\varrho}\right\}_{\varrho \in \mathcal{P}_{1} \cup \mathcal{P}_{3}},\left\{\tau_{\varrho}^{K}\right\}_{\varrho \in \mathcal{P}_{2}}\right)$ is simultaneously cobordant to $\left(K ;\left\{\varepsilon_{\varrho_{0}} \oplus R^{n_{\varrho}-n_{\varrho}}\right\}_{\varrho \in \mathcal{P}_{1}},\left\{\tau_{\varrho}^{K}\right\}_{\varrho \in \mathcal{P}_{2}},\left\{\varepsilon_{\varrho}\right\}_{\varrho \in \mathcal{P}_{3}}\right)$, and $\left(L ;\left\{\mu_{\varrho}\right\}_{\varrho \in \mathcal{P}_{1} \cup \mathcal{P}_{3}},\left\{\tau_{\varrho}^{L}\right\}_{\varrho \in \mathcal{P}_{2}}\right)$ is simultaneously cobordant to $\left(L ;\left\{\mu_{\varrho_{0}} \oplus R^{m_{\varrho}-m_{\varrho}}\right\}_{\varrho \in \mathcal{P}_{1}},\left\{\tau_{\varrho}^{L}\right\}_{\varrho \in \mathcal{P}_{2}},\left\{\mu_{\varrho}\right\}_{\varrho \in \mathcal{P}_{3}}\right)$. 
Now choose $\varrho_{1} \in \mathcal{P}_{1}$ with $n_{\varrho_{1}} \geq n_{\varrho}$ for every $\varrho \in \mathcal{P}_{1}$; then also $m_{\varrho_{1}} \geq m_{\varrho}$ for every $\varrho \in \mathcal{P}_{1}$. Since there is $\varrho \in \mathcal{P}_{1}$ for which $U_{\varrho}=V_{\varrho}$ (that is, with $\left.\operatorname{dim}\left(\mu_{\varrho}\right)>0\right)$, we have $m_{\varrho_{1}}>0$ and thus $U_{\varrho_{1}}=V_{\varrho_{1}}$. For $T \notin \operatorname{ker}\left(\varrho_{1}\right)$, the involution $\left(U_{\varrho_{1}} ; T\right)$ then has fixed data $\left(K ; \varepsilon_{\varrho_{1}}\right) \cup\left(L ; \mu_{\varrho_{1}}\right)$, and thus $\left(U_{\varrho_{1}} ; T\right)$ is equivariantly cobordant to an involution $(W ; T)$ with $W$ connected and with fixed data $\left(K ; \varepsilon_{\varrho_{0}} \oplus R^{n_{\varrho_{1}}-n_{\varrho_{0}}}\right) \cup\left(L ; \mu_{\varrho_{0}} \oplus R^{m_{\varrho_{1}}-m_{\varrho_{0}}}\right)$. The fixed data of the $Z_{2}^{k}$-action $\sigma \Gamma_{r}^{k}(W ; T)$ is then $\left(K ;\left\{\varepsilon_{\varrho}^{\prime}\right\}_{\varrho \in \mathcal{P}_{1}},\left\{\tau_{\varrho}^{K}\right\}_{\varrho \in \mathcal{P}_{2}},\left\{\varepsilon_{\varrho}\right\}_{\varrho \in \mathcal{P}_{3}}\right) \cup$ $\left(L ;\left\{\mu_{\varrho}^{\prime}\right\}_{\varrho \in \mathcal{P}_{1}},\left\{\tau_{\varrho}^{L}\right\}_{\varrho \in \mathcal{P}_{2}},\left\{\mu_{\varrho}\right\}_{\varrho \in \mathcal{P}_{3}}\right)$, where, for each $\varrho \in \mathcal{P}_{1}, \varepsilon_{\varrho}^{\prime}=\varepsilon_{\varrho_{0}} \oplus R^{n_{\varrho_{1}}-n_{\varrho}}$

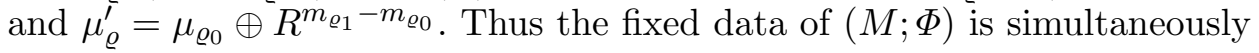
cobordant to a list obtained by removing sections from the fixed data of $\sigma \Gamma_{r}^{k}(W ; T)$, and the theorem is proved.

Examples and final remarks. 1. Let $F$ be the space consisting of $p$ isolated points. It is well known (see [2]) that $\mathcal{A}_{k}(F)=\emptyset$ for $p=1$ and any $k \geq 1$, and $\mathcal{A}_{1}(F)=\emptyset$ for any $p \geq 1$ odd. However, there exist $Z_{2}^{k}$-actions fixing $F$ for each $k \geq 2$ and $p \geq 3$ odd. In fact, consider the $Z_{2}^{k}$-actions $\left(R P^{2} ; T_{1}, \ldots, T_{k}\right)$ and $\left(S^{2} ; T_{1}^{\prime}, \ldots, T_{k}^{\prime}\right)$, where $S^{2} \subset R^{3}$ is the standard 2sphere in the 3 -dimensional euclidean space, $T_{1}\left[x_{0}, x_{1}, x_{2}\right]=\left[-x_{0}, x_{1}, x_{2}\right]$, $T_{2}\left[x_{0}, x_{1}, x_{2}\right]=\left[x_{0},-x_{1}, x_{2}\right], T_{1}^{\prime}(x, y, z)=(-x,-y, z), T_{i}=\operatorname{Id}$ for $i \geq 3$ and $T_{i}^{\prime}=\operatorname{Id}$ for $i \geq 2$. The first action fixes three points and the second fixes two points. Write $p=3+2 t, t \geq 0$. Then the disjoint union of $\left(R P^{2} ; T_{1}, \ldots, T_{k}\right)$ and $t$ copies of $\left(S^{2} ; T_{1}^{\prime}, \ldots, T_{k}^{\prime}\right)$ is a $Z_{2}^{k}$-action fixing $p$ points. This means that $\mathcal{A}_{k}(F) \neq \emptyset$ for $k \geq 2$ and $p \geq 3$ odd. On the other hand, if $p \geq 3$ is odd and $\tau=\left\{\tau_{1}, \ldots, \tau_{s}\right\} \quad(s \geq 1)$ is a partition of the set of components of $F$, then some $\tau_{i_{0}}$ necessarily has an odd number of components. Thus, if $F_{i_{0}}$ is the subspace of $F$ corresponding to $\tau_{i_{0}}$, then $\mathcal{A}_{1}\left(F_{i_{0}}\right)=\emptyset$, and consequently $\mathcal{B}_{k}\left(F_{i_{0}}\right)=\emptyset$ and $\mathcal{P}_{k}(F)=\emptyset$ for every $k \geq 2$.

2. Suppose $F$ is an $n$-dimensional $(n \geq 1)$, connected and closed manifold having Euler characteristic $\chi(F)$ odd (for example, a cartesian product of any number of copies of even-dimensional projective spaces). Let $\eta \rightarrow F$ be any $s$-dimensional vector bundle over $F$ with $s \geq 3$ odd, and let $p: R P(\eta) \rightarrow F$ be the projection map. For $k \geq 2$, consider $E \subset(R P(\eta))^{2^{k}}$, $\left.E=\left\{\left(x_{1}, \ldots, x_{2^{k}}\right) \in R P(\eta)\right)^{2^{k}} \mid p\left(x_{1}\right)=\cdots=p\left(x_{2^{k}}\right)\right\}$. Then $E$ is a closed $\left(n+2^{k}(s-1)\right)$-dimensional submanifold of $(R P(\eta))^{2^{k}}$. On $E$ one has the fiberwise twist $Z_{2}^{k}$-action $(E ; \Phi)$ fixing a diagonal copy of $R P(\eta)$ and with fixed data $\left(R P(\eta) ;\left\{\varepsilon_{\varrho}\right\}_{\varrho \in \mathcal{P}}\right)$, where each $\varepsilon_{\varrho}$ is the $(s-1)$-dimensional vector bundle $\mu \rightarrow R P(\eta)$ tangent along the fiber. Since $R P(\eta)$ is connected, $\mathcal{P}_{k}(R P(\eta))=\mathcal{B}_{k}(R P(\eta))$. Because $\operatorname{dim}\left(\varepsilon_{\varrho}\right)>0$ for every $\varrho$, if $(E ; \Phi) \in$ $\mathcal{B}_{k}(R P(\eta))$ then necessarily $(E ; \Phi)$ is, up to automorphisms of $Z_{2}^{k}$, equivariantly cobordant to $\Gamma_{k}^{k}(W ; T)$ for some involution $(W ; T)$ fixing $R P(\eta)$. But then $\mu \rightarrow R P(\eta)$ is obtained by removing $n$ sections from some $(n+s-1)$ - 
dimensional vector bundle $\theta \rightarrow R P(\eta)$ cobordant to the tangent bundle of $R P(\eta)$. Thus $w_{n+s-1}(\theta)=w_{n+s-1}(R P(\eta))=0$, where $w_{n+s-1}$ denotes the top-dimensional Stiefel-Whitney class. Now if $W(F)=1+w_{1}+\cdots+w_{n}$, $W(\eta)=1+v_{1}+\cdots+v_{s}$ and $W(\xi)=1+c$ are the Stiefel-Whitney classes of $F, \eta$ and the usual line bundle $\xi$, then

$\left.W(R P(\eta))=\left(1+w_{1}+\cdots+w_{n}\right)\left((1+c)^{s}+v_{1}(1+c)^{s-1}+\cdots+v_{s-1}(1+c)+v_{s}\right)\right)$ with $c^{s}+v_{1} c^{s-1}+\cdots+v_{s-1} c+v_{s}=0$. Hence

$$
w_{n+s-1}(R P(\eta))=w_{n}\left(\sum_{t=0}^{s-1}\left(\begin{array}{c}
s-t \\
s-t-1
\end{array}\right) c^{s-t-1} v_{t}\right) .
$$

Because $s$ is odd and for dimensional reasons one then gets $w_{n+s-1}(R P(\eta))$ $=w_{n} c^{s-1}$. Since

$$
w_{n} c^{s-1}[R P(\eta)]=w_{n}[F] \equiv \chi(F) \bmod 2 \quad(\text { see }[2, \text { Lemma 27.2]), }
$$

this gives a contradiction, and $\mathcal{A}_{k}(R P(\eta)) \neq \mathcal{P}_{k}(R P(\eta))$.

3. Together with Theorems 3.9 and 4.2, the explicit determination of $\mathcal{A}_{1}(K \cup L)$ provides an explicit determination of $\mathcal{A}_{k}(K \cup L)$ for every $k \geq 2$. For example, in the recent paper [4], we obtained the cobordism classification of involutions whose fixed point set is $R P^{2} \cup R P^{2 n}$ for every $n>1$. Therefore this gives, up to cobordism, all $Z_{2}^{k}$-actions with this specific fixed set. The details concerning the explicit description of $\mathcal{A}_{k}\left(R P^{2} \cup R P^{2 n}\right)$ for $k \geq 2$ can be seen in [4, pp. 41-43]. As other simple examples, consider $K \cup L=$ $R P^{6} \cup C P^{4}, R P^{2} \cup C P^{4}$ and $C P^{2} \cup R P^{6}$. Denote by $\lambda \rightarrow R P^{n}$ and $\nu \rightarrow C P^{m}$ the canonical real and complex line bundles over $R P^{n}$ and $C P^{m}$, respectively. A routine calculation of characteristic numbers based on [2, Theorem 28.1] shows that $\mathcal{A}_{1}\left(R P^{6} \cup C P^{4}\right)=\emptyset, \mathcal{A}_{1}\left(R P^{2} \cup C P^{4}\right)=\emptyset$ and $\mathcal{A}_{1}\left(C P^{2} \cup R P^{6}\right)=\left\{\left[\left(W^{7} ; T\right)\right],\left[\Gamma\left(W^{7} ; T\right)\right],\left[\Gamma^{2}\left(W^{7} ; T\right)\right]\right\}$, where $\left(W^{7} ; T\right)$ is an involution with fixed data $\left(\nu \oplus R \rightarrow C P^{2}\right) \cup\left(\lambda \rightarrow R P^{6}\right)$ (this means that $\mathcal{A}_{1}\left(C P^{2} \cup R P^{6}\right)$ has only one subset of the type previously described). Thus, up to cobordism, the possible $Z_{2}^{k}$-actions fixing either $R P^{6} \cup C P^{4}$ or $R P^{2} \cup C P^{4}$ are those given by Theorem 3.9; because of the remark after Theorem $3.9, \mathcal{A}_{k}\left(R P^{6} \cup C P^{4}\right)=\emptyset$, and $\mathcal{A}_{k}\left(R P^{2} \cup C P^{4}\right)$ consists of classes whose representatives are of the form $\sigma \Gamma_{r}^{k}\left(R P^{2} \times R P^{2}\right.$; twist $) \cup \sigma^{\prime} \Gamma_{s}^{k}\left(C P^{4} \times C P^{4}\right.$; twist), where $k \geq 2,2 \leq r \leq k$ and $s=r-2$.

Concerning $K \cup L=C P^{2} \cup R P^{6}$, again for dimensional reasons, no action is given by Theorem 3.9. Then every $Z_{2}^{k}$-action fixing $C P^{2} \cup R P^{6}$ is cobordant to a $Z_{2}^{k}$-action obtained by removing sections from the fixed data of an action of the type $\sigma \Gamma_{r}^{k}\left(\Gamma^{2}\left(W^{7} ; T\right)\right)$. For example, up to automorphisms of $Z_{2}^{2}, \mathcal{A}_{2}\left(C P^{2} \cup R P^{6}\right)$ consists of the classes $\left[\Gamma_{i}^{2}\left(\Gamma^{j}\left(W^{7} ; T\right)\right)\right]$, $i=1,2, j=0,1,2$, and three more classes obtained by removing sections from $\Gamma_{2}^{2}\left(\Gamma^{2}\left(W^{7} ; T\right)\right)$ (with fixed data $\left(\nu \oplus R^{3}, \nu \oplus R^{2}, \tau\right) \cup\left(\lambda \oplus R^{2}, \lambda \oplus R, \tau\right)$, 
$\left(\nu \oplus R^{3}, \nu \oplus R, \tau\right) \cup\left(\lambda \oplus R^{2}, \lambda, \tau\right)$ and $\left.\left(\nu \oplus R^{2}, \nu \oplus R, \tau\right) \cup(\lambda \oplus R, \lambda, \tau)\right)$. By applying all the possible automorphisms to these representatives, we obtain a total of 36 classes for $\mathcal{A}_{2}\left(C P^{2} \cup R P^{6}\right)$.

Acknowledgements. We would like to thank Professor Robert E. Stong of the University of Virginia for suggestions and techniques. We also thank Adriana Ramos for a careful reading and helpful suggestions.

\section{References}

[1] F. L. Capobianco, Stationary points of $Z_{2}^{k}$ actions, Proc. Amer. Math. Soc. 61 (1976), 377-380.

[2] P. E. Conner and E. E. Floyd, Differentiable Periodic Maps, Springer, Berlin, 1964.

[3] C. Kosniowski and R. E. Stong, Involutions and characteristic numbers, Topology 17 (1978), 309-330.

[4] R. de Oliveira, P. L. Q. Pergher and A. Ramos, $Z_{2}^{k}$-actions fixing $R P^{2} \cup R P^{e v e n}$, Algebr. Geom. Topology 7 (2007), 29-45.

[5] P. L. Q. Pergher, On $Z_{2}^{k}$ actions, Topology Appl. 117 (2002), 105-112.

[6] -, $Z_{2}^{k}$ actions fixing $\{$ point $\} \cup V^{n}$, Fund. Math. 171 (2002), 83-97.

[7] -,$\left(Z_{2}\right)^{k}$-actions whose fixed data has a section, Trans. Amer. Math. Soc. 353 (2001), 175-189.

[8] P. L. Q. Pergher and R. de Oliveira, $\left(Z_{2}\right)^{k}$-actions with a special fixed point set, Fund. Math. 186 (2005), 97-109.

[9] P. L. Q. Pergher and R. E. Stong, Involutions fixing $\{$ point $\} \cup F^{n}$, Transformation Groups 6 (2001), 78-85.

[10] D. C. Royster, Involutions fixing the disjoint union of two projective spaces, Indiana Univ. Math. J. 29 (1980), 267-276.

[11] R. E. Stong, Equivariant bordism and $\left(Z_{2}\right)^{k}$-actions, Duke Math. J. 37 (1970), 779-785.

[12] —, Involutions fixing projective spaces, Michigan Math. J. 13 (1966), 445-447.

Departamento de Matemática

Universidade Federal de São Carlos

Caixa Postal 676

São Carlos, SP 13565-905, Brazil

E-mail: pergher@dm.ufscar.br
Departamento de Ciências Exatas Universidade Federal de Mato Grosso do Sul Caixa Postal 210

Três Lagoas, MS 79603-011, Brazil E-mail: rogerio@ceul.ufms.br 